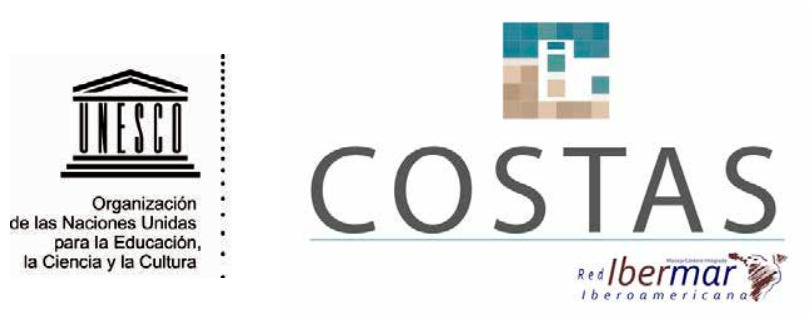

Afanador Franco, F., M.P. Molina Jiménez, L.T. Pusquin Ospina, N. Guevara Cańas, M.J. González Bustillo, K.I. Martínez Uparela, C. Banda Lepesquer, G.A. Escobar Olaya, I. Castro Mercado, 2021 Coastal Marine Planning: Vision of the Maritime Authority.

Case of the Department of Bolivar - Colombia. Revista Costas, vol. esp., 2: 137-164. doi: 10.26359/costas. e0721

Institucional Management Report / Relatórios de Gestão Institucionais / Informe de Gestión Institucionales

Ordenamiento Marino Costero: Visión de Autoridad Marítima. Caso Departamento de Bolivar, Colombia

\title{
Coastal Marine Planning: Vision of the Maritime Authority. Case of the Department of Bolivar, Colombia
}

\author{
Fernando Afanador Franco ${ }^{1}$, Maria P. Molina Jiménez, \\ Lady T. Pusquin Ospina ${ }^{1}$, Natalia Guevara Cañas ${ }^{1}$, María J. González Bustillo ${ }^{1}$, \\ Katia I. Martínez Uparela ${ }^{1}$, Carlos Banda Lepesquer ${ }^{1}$, \\ German A. Escobar Olaya ${ }^{1}$, Ivan Castro Mercado ${ }^{2}$ \\ e-mail: Fernando.Afanador@dimar.mil.co
}

1 Centro de Investigaciones Oceanográficas e Hidrográficas del Caribe (CIOH). Área de Manejo Integrado de Zonas Costeras. Barrio El Bosque, Isla de Manzanillo, Escuela Naval "Almirante Padilla" Cartagena de Indias, Bolívar, Colombia.

${ }^{2}$ Dirección General Marítima. Subdirección de Desarrollo Marítimo. Litorales y Áreas Marinas. Carrera 54 \# 26-50, Bogotá, Colombia.

Submitted: October 2020

Accepted: February 2021

Associate Editor: Alejandro Iglesias-Campos, Michele Quesada-Silva y Marinez Scherer
Keywords: Marine Spatial Planning, Conflict Analysis, Geographic Information System, Use, Multicriteria evaluation.

\begin{abstract}
Marine Spatial Planning is a tool that has acquired significant importance worldwide. Around 70 countries have implemented this initiative given the increased activity within the maritime sector and pressure on marine resources. The methods used are adapted to each country's characteristics and articulated with other management processes. Although Colombia has progressed through on the processes regarding this issue, through different agencies, marine spatial planning related to maritime activities is absent. Therefore, the General Maritime Directorate (DIMAR in Spanish) through its commitment to turning Colombia into a bi-oceanic power, under a holistic and
\end{abstract}




\section{COSTAS}

Revista Costas, Vol. Esp. 2, 2021

comprehensive maritime safety approach, contributes to marine and coastal areas management with a methodology for Marine and Coastal Management with a Maritime Authority Vision (MCM: MAV), focused on analyzed current and future conditions using Geographic Information Systems (GIS), multi-criteria analysis, and an Allocation and Co-location Model (ACM). The method was applied to Bolivar Department marine and coastal area, resulting in the identification of 55 uses/activities, and obtaining zoning by index and by the number of conflicts, as well as a map of free areas. This information is intended to improve monitoring, evaluation, and updating of maritime activities in these areas, and because it is applicable throughout the Colombian territory, it facilitates decision-making by several national governmental agencies.

\section{Resumen}

La Planificación Espacial Marina es una herramienta que ha tomado gran importancia a nivel mundial. Alrededor de 70 países han implementado esta iniciativa debido a la creciente actividad en el sector marítimo y a la presión sobre los recursos marinos. Las metodologías aplicadas se ajustan a las características propias de cada país y se pueden articular con otros procesos de ordenamiento. En Colombia aunque se han adelantado procesos en este tema a través de diferentes entidades, no se ha generado un ordenamiento espacial de los usos relacionados con las actividades marítimas. Es por esta razón que la Dirección General Marítima (DIMAR) con su compromiso de convertir a Colombia en una potencia bioceánica, bajo un enfoque holístico y de seguridad integral marítima, realiza su aporte a la gestión de los espacios marino - costeros a través de una metodología de Ordenamiento Marino Costero con una Visión de Autoridad Marítima (OMC: VAM), en la cual se busca analizar las condiciones actuales y futuras empleando Sistemas de Información Geográfica (SIG), análisis multicriterio y un Modelo de Asignación y Co-localización (MAYC). La metodología se aplicó en la zona marino - costera del departamento de Bolívar identificando 55 usos/actividades, obteniendo la zonificación por índice y número de conflictos y el mapa de zonas libres. Con esta información se pretende mejorar el seguimiento, evaluación y actualización de las actividades marítimas en estas áreas, y al ser aplicable en todo el territorio colombiano, facilita la toma de decisiones de las diferentes entidades gubernamentales del país.

Palabras clave: Planificación Espacial Marina, Análisis de conflictos, Sistemas de Información Geográfica, usos, evaluación multicriterio.

\section{Introducción}

Se estima que más de 3 billones de personas a nivel mundial dependen de los océanos ya que contribuyen al desarrollo de diferentes sectores económicos y además son fuente de alimento y empleos directos e indirectos (FAO, 2014; Prato \& Reyna, 2015; Papadimitriou et al., 2019; OECD, 2020). Sin embargo, actividades que involucran la interfaz tierra-mar con alto potencial de crecimiento en las áreas marino costeras, como el tráfico marítimo, la energía marina renovable, la pesca, la acuicultura, plataformas off shore, el desarrollo de la infraestructura costera, entre otras, han intensificado la presión sobre los recursos marinos y han generado una serie de problemáticas y conflictos de uso entre los diferentes actores que aprovechan estas áreas ya que en algunas ocasiones estas actividades se superponen causando diferentes efectos que pueden llevar a un uso insostenible de los mismos (Cicin-Sain \& Knecht, 1998; MarViva, 2013; EP\&C, 2013; Christie et al., 2014; Portman et al., 2015; Tsilimigkas \& Rempis 2017).

Estas situaciones se aumentan como consecuencia de la falta de aplicación de políticas adecuadas para el ordenamiento y la dificultad para resolver conflictos entre usuarios y el ambiente (Aldana \& Hernández, 2016). Sin embargo, a través del enfoque de interacciones tierra- mar (LSI, por sus siglas en inglés) se 


\section{re \\ COSTAS}

busca promover el uso sostenible del espacio marítimo, mediante interrelaciones entre actividades socioeconómicas y acuerdos de gobernanza que aseguren la integridad del hábitat para vivir, funcionar y proporcionar muchos de los servicios ecosistémicos de los que dependen los humanos (Inniss et al., 2016; Portman et al., 2015; Millennium Ecosystem Assessment, 2005).

Las zonas marino - costeras debido a sus características y condiciones multidimensionales, en la que se involucra el fondo marino, la columna de agua y la superficie misma (Douvere, 2010; Bonnevieet al., 2019) están abiertas a diferentes puntos de vista desde varias disciplinas y áreas de conocimiento en los que se involucran aspectos sociales, económicos, ambientales y políticos (Ansong et al., 2019; Ehler et al. 2019). Colombia, es el único país de Suramérica con acceso a los dos océanos, Pacífico y Atlántico (Mar Caribe), razón por la cual no es ajena a la diversidad de actividades marítimas y a las implicaciones por su desarrollo (DNP, 2020). El espacio marino-costero del departamento de Bolívar, es una zona de convergencia de varios usos/actividades, debido a que su capital la ciudad de Cartagena de Indias, es considerada el principal destino turístico del país, así como la puerta de entrada del comercio, debido al desarrollo portuario favorecido por su cercanía a las rutas de comercio marítimo (canal de Panamá), que la ubican dentro del mayor nodo de conectividad en el mundo. Sumado a lo anterior es una de las ciudades más importantes en el sector astillero, por tener tres de las empresas más grandes de este sector; es la cuarta ciudad industrial del país, sede de la segunda refinería de petróleo más importante de Colombia y principal exportador de sustancias químicas (Agencia de inversiones de Cartagena de Indias y Bolívar ,2012; Martínez \& Malagón, 2014; CEDEC \& CCC, 2018).

La Autoridad Marítima Colombiana como responsable de la ejecución, regulación y coordinación de las actividades marítimas establecidas en el Decreto Ley $N^{\circ}$ 2324/1984 (Ministerio de Defensa Nacional 1984), ha ejercido funciones sobre los litorales desde antes de los años 80 's, y al igual que otras entidades del orden nacional ha contribuido al ordenamiento marino - costero del país de una manera integral y holística (Steer et al., 1997; CCO, 2018). A pesar de lo anterior, no existe un ordenamiento eficiente de los usos/actividades del territorio marino - costero (Franco Herrera et al., 2020).

En países como Alemania, China, Australia, Países Bajos, Indonesia, Bélgica, Uruguay, entre otros, incluyendo Colombia, los estudios de planificación y ordenamiento para la gestión del espacio marino costero, se ajustan a las particularidades de cada uno de ellos y a los objetivos de la economía azul, para un desarrollo sostenible de los ambientes marinos, al equilibrar aspectos ambientales, económicos y sociales que reduzcan los conflictos de uso y proporcionen apoyo a los formuladores de políticas (UNEP, 2015; Jones et al., 2016; UNESCO, 2017; Frazão et al., 2019; Fang et al., 2019; European Commission, 2019, 2020).

Teniendo en cuenta la necesidad de establecer un ordenamiento de las actividades marítimas, la Dirección General Marítima (DIMAR), desde el Centro de Investigaciones Oceanográficas e Hidrográficas del Caribe (CIOH), estableció la metodología de Ordenamiento Marino Costero, con una Visión de Autoridad Marítima (OMC:VAM) con el objetivo de analizar y asignar distribuciones temporales y espaciales de actividades humanas en aguas jurisdiccionales y zonas costeras colombianas, en busca de lograr la consolidación del país como una potencia bioceánica, bajo un enfoque holístico y de seguridad integral marítima, fluvial y portuaria; así como también, garantizando los principios ecológicos, económicos y sociales (DIMAR, 2018, 2019a; DNP, 2020). 


\section{rig \\ COSTAS}

Este artículo está basado en la publicación denominada "Conflictos de uso en el proceso de Ordenamiento Marino Costero: Visión de Autoridad Marítima. Departamento de Bolívar - Colombia”

\section{2. Área de estudio}

Ubicada en la región Caribe colombiana, el litoral del departamento de Bolívar entre el municipio de Galerazamba y el sector de Caño Matunilla (figura 1), abarca una extensión de $529.86 \mathrm{Km}$ de línea de costa y cubre aproximadamente $47333.4740 \mathrm{~km}^{2}$ divididos entre Zona A (definida desde el límite de los bienes de uso público establecido por la DIMAR hasta las 12 millas náuticas) y Zona B (desde las 12 hasta las 200 millas náuticas). publicado en la revista Boletín Científico $\mathrm{CIOH}$ de la Dirección General Marítima de Colombia (DIMAR), y presenta como resultados la actualización del proceso metodológico del OMC:VAM.
En esta región, los patrones de viento, oleaje y clima dependen de la dinámica de los vientos alisios del NE, influenciada a su vez por la Zona de Convergencia Intertropical (ZCIT) (Leblé and Cuignon 1987). La posición meridional y septentrional de la ZICT indican estación seca de diciembre a abril y húmeda de agosto a noviembre respectivamente. De mayo a julio hay una transición entre estas dos estaciones (Pujos et al., 1986; Andrade-Amaya, 2001).

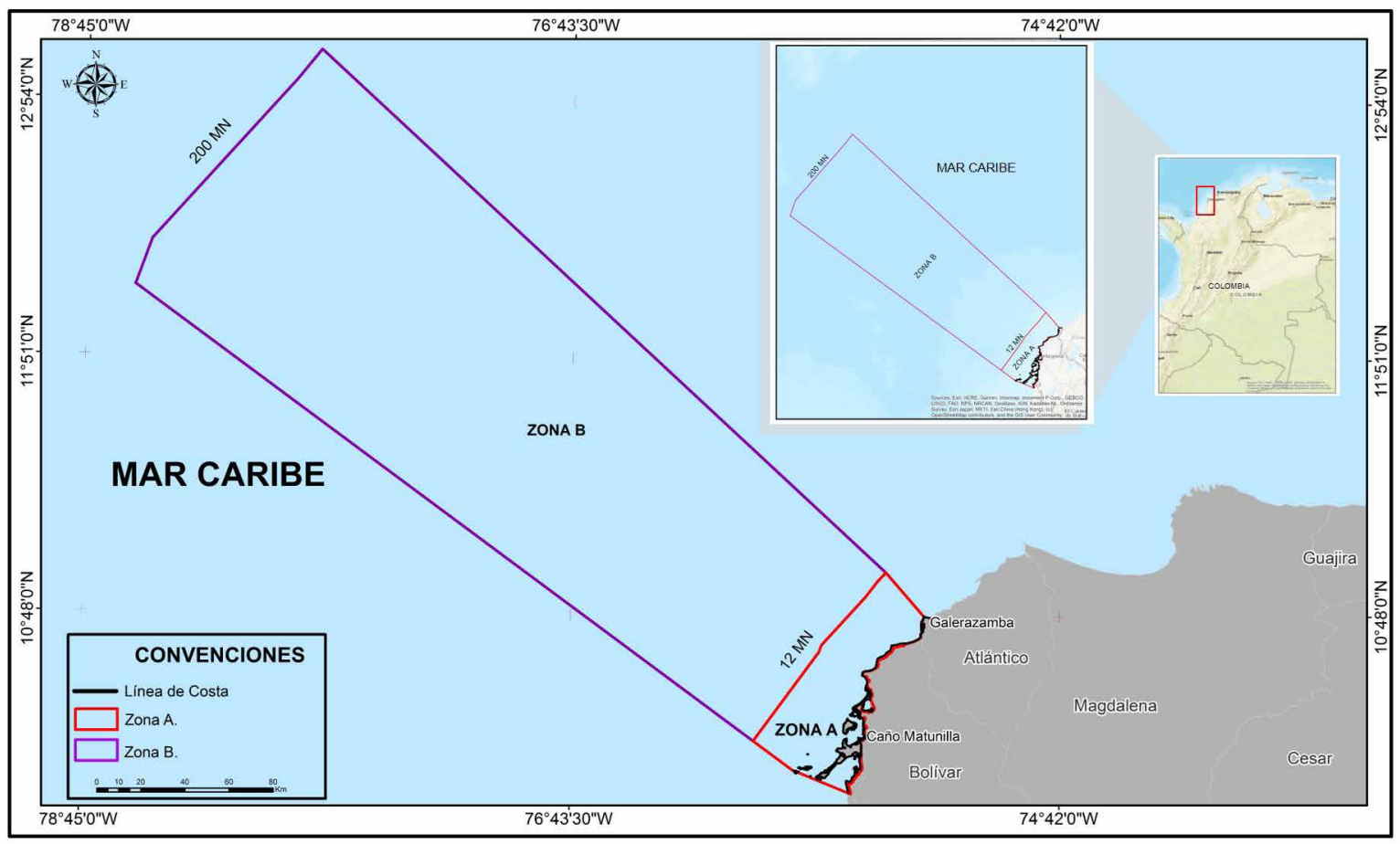

Figura 1. Localización del área de estudio en el Caribe Colombiano.

Figure 1. Study area in the Colombian Caribbean. 
Afanador Franco et al.

En el departamento de Bolívar, la mayor concentración de manglares se encuentra hacia la zona sur, asociados a ciénagas, zonas de inundación, depósitos aluviales del canal del Dique y en la bahía de Cartagena a manera de parches. Se reportan cinco especies: Rhizophora mangle, Avicennia germinans, Laguncularia racemosa, Conocarpus erecta y Pelliciera rhizophorae (CARDIQUE 1998; Carvajal and Jurado 2009). De igual forma la mayor concentración de formaciones coralinas están reportadas en el Parque Nacional Natural Corales del Rosario y de San Bernardo, con un área total aproximada de $358.6 \mathrm{Km}^{2}$ que corresponden a cobertura de arrecifes franjeantes, de parche, tapetes y bancos coralinos (Díaz et al., 2000; Alvarado et al., 2011).

\section{Metodología}

La metodología de OMC: VAM toma en consideración que los espacios marinos, costeros e insulares son ambientes dinámicos donde coexisten diferentes usos que generan conflictos y causan efectos en el ambiente (Cicin-Sain \& Knetch, 1998; Douvere et al., 2007; Tsilimigkas \& Rempis, 2017). Con su desarrollo y aplicación se busca la disminución de los conflictos entre usos/usuarios, la conservación de la base natural, la equidad en el desarrollo económico y en la distribución del espacio (Gilliland \& Laffoley, 2008; Tsilimigkas \& Rempis, 2017; DIMAR ,2019a).

El OMC: VAM se aplicó en el área marino - costera del departamento de Bolívar (Colombia) siguiendo seis etapas (figura 2).

\section{Establecimiento de los elementos de gobernanza.}

La gestión de los espacios marinos, costeros e insulares aplica el concepto de gobernanza, ya que se hace necesaria la interacción entre actores públicos y

\section{re \\ COSTAS}

El Caribe colombiano se caracteriza por tener un ambiente tectónico compresional debido a la interacción entre las placas Suramericana, Caribe y Nazca (Trenkamp et al., 2002; Audemard and Audemard 2002). En general en el área de estudio se pueden encontrar rocas sedimentarias de origen marino profundo a litoral, como Areniscas líticas y de Cuarzo, Gravas, Calizas Arrecifales, Lodolitas, Depósitos Coluviales, de Playa y de Llanura Costera y rasgos morfológicos de origen estructural, denudacional y litoral como cuestas, depósitos de playa, piedemontes (INGEOMINAS, 2001), colinas, terrazas marinas, espigas, barras, llanuras costeras, ciénagas, dunas, planicie aluvial, llanuras de inundación, entre otros (Carvajal \& Jurado, 2009).

privados, teniendo en cuenta sus diversos intereses y funciones con el compromiso de lograr un desarrollo socio-económico que permita un equilibrio entre la sociedad, las entidades reguladoras, la economía y el ecosistema (Zervaki, 2018; CCO, 2018). El OMC: VAM define la prioridad estratégica nacional a partir de la identificación de las políticas, convenios y/o acuerdos nacionales e internacionales que proporcionen lineamientos para el Ordenamiento Marino Costero en el marco de la Visión de Autoridad Marítima; las escalas cartográficas, para facilitar la representación y visualización de la información; la base cartográfica y la documentación espacial y fotográfica de los diferentes usos en la zona de interés.

\section{Etapa de pre-planeación}

Busca identificar los diferentes actores involucrados en el OMC: VAM del departamento de Bolívar, establecer las categorías de uso y definir los objetivos para la resolución de conflictos. 


\section{COSTAS}

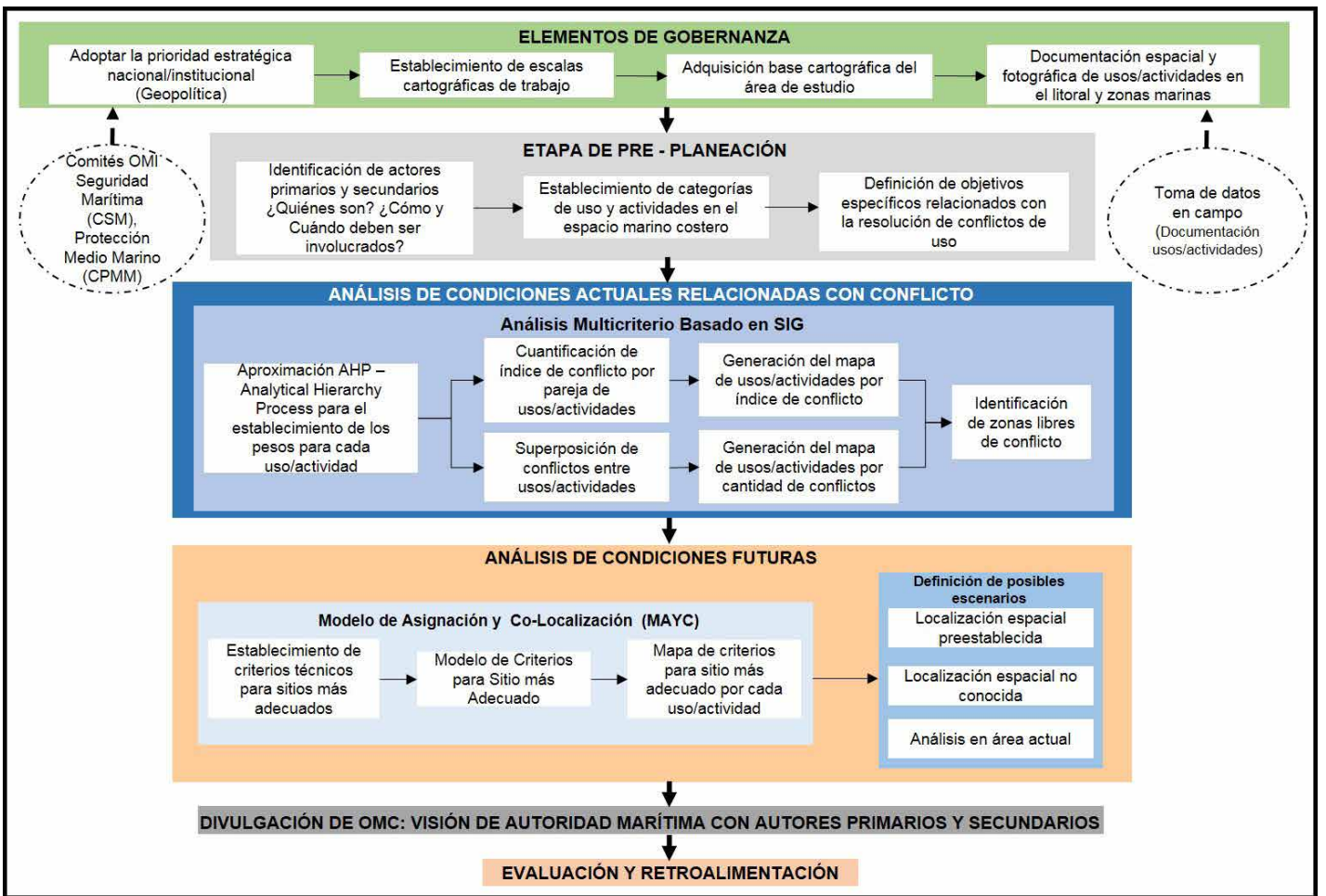

Figura 2. Etapas para la aplicación del OMC: VAM (DIMAR 2019a).

Figure 2. MCM: MAV stages of implementation (DIMAR 2019a).

Los actores relacionados con las actividades marítimas, se clasificaron en primarios que corresponden a aquellos que aprovechan directamente el recurso y contribuyen al conflicto, y secundarios que son las entidades estatales que controlan los usos que se desarrollan en los espacios marinos, costeros e insulares (Maguire et al., 2012).

Teniendo en cuenta las actividades realizadas por los diferentes actores se establecen las categorías de uso basadas en las actividades marítimas que se estipulan en el Decreto Ley 2324 de 1984 que establece las funciones de la Autoridad Marítima Colombiana (Ministerio de Defensa Nacional 1984) (tabla 1), la clasificación de la base ecosistémica de acuerdo con de Groot et al. (2002), Portman (2016) e información de los Ecosistemas Continentales, Costeros y
Marinos del Instituto de Hidrología, Meteorología y Estudios Ambientales (IDEAM et al., 2017) (tabla 2).

\section{Análisis de condiciones actuales relacionadas con conflictos}

El objetivo principal de esta etapa es establecer las compatibilidades e incompatibilidades existentes entre los usos y actividades marítimas y la base ecosistémica, empleando el análisis multicriterio basado en Sistemas de Información Geográfica (SIG), con una aproximación AHP (Analytical Hierarchy Process) para cuantificar a través del juicio de expertos y de técnicas de optimización espacial los conflictos entre usos y establecer las zonas con conflictos, zonas libres de conflictos y áreas disponibles para futuros 


\begin{tabular}{|c|c|}
\hline Ítem & Actividades Marítimas \\
\hline 1 & Señalización marítima. \\
\hline 2 & Control del tráfico marítimo. \\
\hline 3 & Naves nacionales y extranjeras y los artefactos navales \\
\hline 4 & La navegación marítima por naves y artefactos navales \\
\hline 5 & Marina mercante y el transporte marítimo \\
\hline 6 & Comunicaciones marítimas \\
\hline 7 & Construcción, operación y administración de instalaciones portuarias \\
\hline 8 & Utilización, protección y preservación de los litorales \\
\hline 9 & Investigación científica marina en todas sus disciplinas \\
\hline 10 & Sistemas de exploración, explotación y prospección de los recursos naturales del medio marino \\
\hline 11 & Búsqueda y extracción o recuperación de antigüedades o tesoros náufragos \\
\hline 12 & Recreación y el deporte náutico marinos. \\
\hline 13 & Búsqueda y salvamento marítimos. \\
\hline 14 & Conservación, preservación y protección del medio marino. \\
\hline 15 & Colocación de cualquier tipo de estructuras, obras fijas o semifijas en el suelo o en subsuelo marinos \\
\hline 16 & Servicio de pronósticos de mar y de tiempo \\
\hline 17 & Rellenos, dragados y obras de ingeniería oceánica. \\
\hline 18 & Administración y desarrollo de la zona costera \\
\hline 19 & Astilleros y la construcción naval \\
\hline 20 & Otros usos y/o aprovechamiento marino. \\
\hline
\end{tabular}

usos (Malczewski, 1999; Brown et al., 2001; Villa et al., 2002; Kennedy et al., 2008; Regan et al., 2007; Yatsalo et al., 2007; Stelzenmüller et al., 2013).

\section{Análisis de condiciones futuras}

El Ordenamiento Marino Costero bajo la premisa de la economía azul, considera las zonas de desarrollo local, regional y nacional, en las cuales es posible llevar a cabo actividades tradicionales y nuevas, buscando reducir los riesgos ambientales y daños ecológicos (Lester et al., 2013; UNEP, 2015; Schultz-Zehden et al., 2019). Por esta razón es importante identificar los posibles conflictos y así considerar escenarios futuros que faciliten la toma de decisiones (Coccoli et al., 2018).
De acuerdo con lo anterior esta etapa busca identificar los sitios más adecuados para la ubicación de nuevos usos/actividades, mediante la aplicación de un Modelo de Asignación y Co-localización (MAYC), que contempla tres escenarios que se pueden presentar ante la Autoridad Marítima colombiana: i) Localización espacial conocida para un uso determinado, ii) localización espacial no conocida de un uso determinado y iii) localización de un uso determinado en el área actual (DIMAR, 2019a).

El análisis de Asignación busca establecer con base en criterios técnicos y ambientales, los sitios más adecuados para la ubicación y desarrollo de diferentes usos/actividades con el objetivo de realizar una zonificación óptima de los usos en la zona marino - 


\section{Fin \\ COSTAS}

Tabla 2. Clasificación ecosistémica de acuerdo a de Groot et al. (2002) y Portman (2016).

Table 2. Ecosystems classification according to de Groot et al. (2002) and Portman (2016).

\begin{tabular}{|l|l|l|}
\hline $\begin{array}{c}\text { Tipo de Servicio } \\
\text { Ecosistémico }\end{array}$ & \multicolumn{1}{|c|}{ Explicación } & \multicolumn{1}{|c|}{ Ejemplo } \\
\hline Hábitat & $\begin{array}{l}\text { Provisión de condiciones espaciales para el man- } \\
\text { tenimiento de la biodiversidad. }\end{array}$ & $\begin{array}{l}\text { Zonas de registros biológicos } \\
\text { Zonas de concentración de especies } \\
\text { Zonas de diversidad biológica } \\
\text { Zonas de anidación de especies }\end{array}$ \\
\hline Producción & $\begin{array}{l}\text { Capacidad de los ecosistemas para crear biomasa } \\
\text { que pueda usarse como alimento, tejidos, etc. }\end{array}$ & $\begin{array}{l}\text { Zonas de alimento (pesca, caza, recolección de frutos, } \\
\text { etc.) } \\
\text { Zonas de recursos forestales maderables y no maderables } \\
\text { Plantas medicinales }\end{array}$ \\
\hline Información & $\begin{array}{l}\text { Capacidad de los ecosistemas de contribuir al } \\
\text { bienestar humano a través del conocimiento, } \\
\text { la experiencia, y las relaciones culturales con la } \\
\text { naturaleza }\end{array}$ & $\begin{array}{l}\text { Zonas de Ecoturismo } \\
\text { Zonas de valores estéticos y paisajísticos }\end{array}$ \\
\hline Regulación & $\begin{array}{l}\text { Procesos relacionados con la capacidad de los } \\
\text { ecosistemas naturales y seminaturales para regu- } \\
\text { lar los procesos ecológicos esenciales. }\end{array}$ & $\begin{array}{l}\text { Zonas de regulación hídrica } \\
\text { Zona de formación de suelo } \\
\text { Zonas de retención de nutrientes y dilución de } \\
\text { contaminantes } \\
\text { Zonas de control biológico }\end{array}$ \\
\hline
\end{tabular}

costera (Farahani \& Hekmatfar 2009; Coccoli et al., 2018; Afanador-Franco et al., 2019).

El análisis de Co-localización permite identificar la localización más adecuada para futuros usos en zonas donde ya se desarrollan actividades, analizando criterios de compatibilidad e incompatibilidad con el propósito de reducir los conflictos al nivel más bajo posible (Hennessey and Sutinen 2005; Marine Management Organization 2013) (tabla 3).

Los resultados de la aplicación del análisis de Asignación y Co-localización se encuentran en desarrollo y serán presentados en una próxima publicación.

\section{Divulgación de OMC:}

\section{Visión de Autoridad Marítima}

\section{con actores primarios y secundarios}

El objetivo de esta etapa es dar a conocer el OMC: VAM, diseñando herramientas y técnicas de comu- nicación para que los actores primarios y secundarios comprendan por una parte la metodología y sus resultados, y por otra realicen sus aportes a partir de la coordinación intrainstitucional y con las comunidades, que facilite el desarrollo del proceso en el ordenamiento de los espacios marino - costeros (Manwaring \& Orenstein 2011).

\section{Evaluación y retroalimentación}

Esta etapa consiste en realizar el seguimiento y evaluación de la metodología aplicada a través del establecimiento de un sistema de monitoreo con indicadores de ejecución y con capacidad para la generación de informes requeridos por los tomadores de decisiones y actores involucrados (DIMAR, 2019a). 
Tabla 3. Escala de compatibilidad e incompatibilidad entre par de usos/actividades (DIMAR 2019a). Table 3. Scale of compatibility and incompatibility of pairs of uses/activities (DIMAR 2019a).

\begin{tabular}{|l|c|l|}
\hline \multicolumn{1}{|c|}{ Criterios de compatibilidad } & Valor & \multicolumn{1}{|c|}{ Expresión de la compatibilidad/ incompatibilidad } \\
\hline Los dos usos se mejoran mutuamente & 1.0 & Altamente compatible \\
\hline Los usos no interfieren el uno con el otro & 0.75 & Compatible \\
\hline Un uso puede mejorar al otro & 0.5 & Condicionalmente compatible \\
\hline Uno de los usos afecta negativamente al otro & 0.25 & Condicionalmente incompatible \\
\hline Los dos usos se afectan negativamente el uno al otro & 0.0 & Incompatible \\
\hline
\end{tabular}

\section{Resultados}

\section{Establecimiento de los elementos de gobernanza}

\section{Adopción de la prioridad estratégica nacional}

El OMC: VAM a nivel nacional se enmarca en lo establecido en el Plan Nacional de Desarrollo con los pactos de: Legalidad, Equidad, Sostenibilidad, Descentralización y Productividad y equidad en las regiones, buscando la protección de los bienes públicos, igualdad de oportunidades, equilibrio entre la conservación y la producción, gobernanza multinivel y participación en escenarios internacionales relacionados con los océanos (Departamento Nacional de Planeación, 2019).

De igual forma la Política Nacional del Océano y de los Espacios Costeros (PNOEC) y el Consejo Nacional de Política Económica y Social 3990, estipulan el objetivo interinstitucional de convertir a Colombia en una potencia bioceánica a través de la conexión del territorio continental al territorio oceánico, procedimientos y protocolos para la gestión del riesgo y sus efectos en la población y sus actividades, administración territorial en los espacios marino-costeros, generación de conciencia marítima en la población nacional, mantenimiento de la cooperación marítima con los Estados aliados y proyectos que permitan reforzar y mejorar los aspectos débiles en temas oceánicos (CCO, 2018; DNP, 2020).
La DIMAR como Autoridad Marítima, complementa esta prioridad estratégica nacional con los denominados "faros institucionales", velando por la seguridad en las operaciones y actividades marítimas, minimizando los riesgos de siniestros e incidentes que atenten contra la vida humana en el mar y garantizando el éxito y la sostenibilidad de las actividades, a través del concepto de Seguridad Integral Marítima (DIMAR, 2018), lo cual implica controlar los procedimientos en las actividades y educar profesionales en aspectos marítimos, con el objetivo de cumplir con las regulaciones establecidas (Bueger, 2015).

\section{Establecimiento de escalas cartográficas de trabajo}

La representación cartográfica de los usos/actividades identificados en el área de estudio se planteó a una escala comprendida entre 1:25000 y 1:50000 para la zona $A$, y una escala entre 1:50000 y 1:200000 para la zona $\mathrm{B}$, con el objetivo de visualizar de una manera correcta cada uno de los resultados temáticos.

\section{Base cartográfica del área de estudio}

La base cartográfica recopila información de diferentes fuentes como Infraestructura de Datos Espaciales (IDE) Marítima, Fluvial y Costera de Colombia antes SIG-DIMAR, Sección de Náutica (del Servicio Hidrográfico Nacional), Agencia Nacional de Hidro- 


\section{ren COSTAS}

carburos (ANH), Plan de Ordenamiento Territorial de Cartagena, Tremarctos Colombia 3.0, Parques Nacionales Naturales de Colombia, Sistema de Información para la Gestión de los Manglares de Colombia (SIGMA) y Centro de Investigaciones Oceanográficas e Hidrográficas del Caribe $(\mathrm{CIOH})$. Para la zona marino - costera del departamento de Bolívar se estructuró la base cartográfica (Geodatabase) con un total de 55 usos/actividades entre actividades marítimas, usos del suelo y base ecosistémica (Afanador-Franco et al., 2019).

\section{Documentación espacial y fotográfica de usos/actividades en el litoral y zonas marinas}

La documentación espacial y fotográfica de usos/actividades tiene como objetivo complementar la base cartográfica del área de estudio. Se llevó a cabo por medio de recorridos en la zona marino - costera del departamento de Bolívar con un total de 120.98 km de línea de costa, en los cuales se diligenció un formulario instalado en dispositivos móviles que contiene los campos de ubicación geográfica, observaciones y fotografías para cada uno de los 375 usos/actividades identificados. El mayor registro documentado fue vegetación abierta sin uso establecido, con un 23.47 $\%$ del total de usos (figura 3).
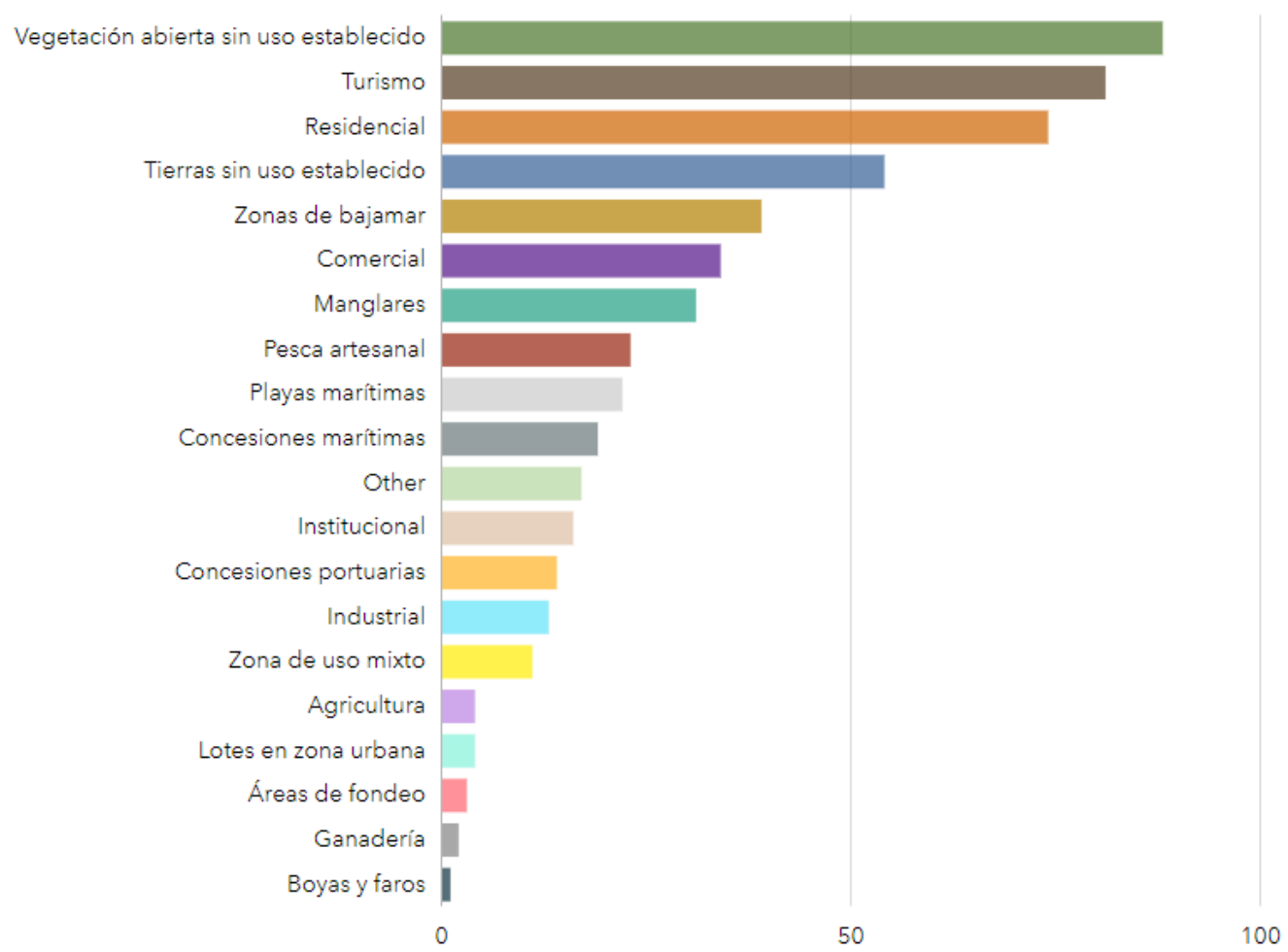

Figura 3. Porcentaje de registros de la documentación espacial y fotográfica de usos/actividades en la zona marino - costera del departamento de Bolívar.

Figure 3. Percentage records of spatial and photographic documents on uses / activities in Bolivar Department marine and coastal areas. 


\section{re \\ COSTAS}

\section{Etapa de pre-planeación}

Identificación de actores primarios y secundarios. El departamento de Bolívar, es una zona donde confluyen diversos usos/actividades, con diferentes misiones, visiones, objetivos y formas de ser constituidas, que implica una cantidad considerable de actores que hacen uso y/o controlan el espacio marino - costero (Gopnik et al., 2012; Martínez \& Malagón, 2014; CEDEC \& CCC, 2018).

Con base en lo anterior se respondieron las siguientes preguntas:

¿Quiénes deben ser involucrados en el proceso? Se identificaron todas las personas, instituciones y entidades que realizan actividades o tienen jurisdicción en el espacio marino - costero del departamento de Bolívar. Como resultado, se establecieron 512 actores primarios, entre los que se encuentran operadores turísticos, pescadores artesanales e industriales, concesiones marítimas y portuarias, transporte marítimo, entre otros; y 22 actores secundarios, de los sectores defensa, ambiental, hidrocarburos, tierras, turismo, industria, pesca y telecomunicaciones (Afanador-Franco et al., 2019).

¿Cómo deben ser involucrados? La divulgación de los resultados obtenidos en el OMC: VAM, se realizará con el apoyo de profesionales en ciencias sociales y jurídicas, a través de la implementación de una metodología de intervención social con actores primarios y secundarios (Afanador-Franco et al., 2019).

¿Cuándo deben ser convocados al proceso? Los actores identificados estarán involucrados en la etapa de divulgación de los resultados, con el propósito de que conozcan el proceso de OMC: VAM y aporten sus ideas para el fortalecimiento del mismo, las cuales serán tomadas en consideración durante la etapa de evaluación y retroalimentación (Afanador-Franco et al., 2019).

\section{Establecimiento de categorías de uso $y$ actividades en el espacio marino-costero.}

A partir de la información de usos/actividades obtenida de las diferentes fuentes, se ordenaron 41 usos en 12 categorías de actividades marítimas, entre las cuales se incluyó la base ecosistémica según la función de los ecosistemas de hábitat y producción, y 14 usos incluidos en la clasificación de usos del suelo (tabla 4).

\section{Objetivos específicos relacionados} con la resolución de conflictos

Los objetivos para la resolución de conflictos son: i) Minimizar los conflictos existentes entre usos/actividades en la zona marino - costera del departamento de Bolívar al nivel más bajo posible y ii) asignar de manera óptima la localización de usos/actividades dentro del espacio geográfico correspondiente (Gilliland \& Laffoley, 2008; DIMAR, 2019b; Afanador-Franco et al., 2019).

\section{Análisis de condiciones actuales relacionadas con conflictos}

\section{Análisis multicriterio basado en SIG}

A partir del análisis multicriterio se define la relación existente entre cada uso/actividad a través de una escala única de intensidad de importancia, permitiendo así, la gestión en diferentes procesos relacionados con las actividades marítimas, al integrar herramientas de Sistemas de Información Geográfica y el juicio de expertos con la técnica del grupo nominal que permite la interpretación subjetiva y cualitativa entre expertos, en un ambiente controlado (Malczewski, 1999; Ouchi, 2004; Kennedy et al., 2008; Stelzenmüller et al., 2013). 
Tabla 4. Usos/actividades identificadas en la zona marino - costera del departamento de Bolívar. Table 4. Uses/activities identified in the Bolivar Department marine and coastal area.

\begin{tabular}{|c|c|c|}
\hline $\begin{array}{l}\text { Actividades Marítimas } \\
\text { (Decreto Ley 2324/84) }\end{array}$ & Usos/ Actividades & Subdivisión de Uso/Actividad \\
\hline \multirow{5}{*}{$\begin{array}{l}\text { Conservación, preservación } \\
\text { y protección del medio marino }\end{array}$} & Concentración de Especies & $\begin{array}{l}\text { Grandes grupos Caribe. } \\
\text { Sitios de registros bilógicos. } \\
\text { Zonas de concentración de especies } \\
\text { amenazadas. } \\
\text { Zonas de concentración de anfibios. } \\
\text { Zonas de concentración de aves. } \\
\text { Zonas de concentración de mamíferos. } \\
\text { Zonas de concentración de reptiles. } \\
\text { Zonas de concentración de cocodrilos. }\end{array}$ \\
\hline & $\begin{array}{l}\text { Anidación y Forrajeo de tortu- } \\
\text { gas marinas }\end{array}$ & $\begin{array}{l}\text { Puntos de anidación de tortugas mari- } \\
\text { nas. } \\
\text { Zonas de anidación de tortugas marinas. } \\
\text { Zonas de forrajeo de tortugas marinas. }\end{array}$ \\
\hline & Desove de langostas & Desove de langostas \\
\hline & Áreas de Conservación & $\begin{array}{l}\text { Áreas significativas de biodiversidad. } \\
\text { Sitios de conservación de la plataforma. } \\
\text { Parques Nacionales Naturales. } \\
\text { Área Marina Protegida (AMP). }\end{array}$ \\
\hline & Pesca & $\begin{array}{l}\text { Zona pesca camarón agua somera. } \\
\text { Pesca artesanal. } \\
\text { Pesca atún Caribe. } \\
\text { Ruta de pesca camarón de aguas } \\
\text { someras. } \\
\text { Ruta de pesca atunera. }\end{array}$ \\
\hline \multirow[t]{2}{*}{ Utilización, protección y preservación de los litorales } & Bienes de Uso Público & $\begin{array}{l}\text { Playas. } \\
\text { Bajamares. }\end{array}$ \\
\hline & Manglares & Manglares. \\
\hline Navegación maritima por naves y artefactos navales & $\begin{array}{l}\text { Navegación Marítima por naves } \\
\text { y artefactos navales }\end{array}$ & Zonas de ejercicios navales. \\
\hline Búsqueda y extracción o recuperación de antigüedades & Naufragios & Naufragios. \\
\hline Recreación y deporte náutico marinos & Buceo & Buceo. \\
\hline Astilleros y construcción naval & Astilleros y construcción naval & Astilleros y construcción naval. \\
\hline Rellenos, dragados y obras de ingeniería oceánica & $\begin{array}{l}\text { Rellenos, dragados y obras de } \\
\text { ingeniería oceánica }\end{array}$ & $\begin{array}{l}\text { Rellenos, dragados y obras de ingeniería } \\
\text { oceánica. Obras de protección costera. }\end{array}$ \\
\hline $\begin{array}{l}\text { Colocación de cualquier tipo de estructuras, obras fijas } \\
\text { o semifijas en el suelo o en el subsuelo marinos }\end{array}$ & Cables submarinos & Cables submarinos. \\
\hline Señalización maritima & Boyas y faros & Boyas y faros. \\
\hline $\begin{array}{l}\text { Construcción, operación y administración } \\
\text { de instalaciones portuarias }\end{array}$ & Concesiones portuarias & Concesiones Portuarias Marítimas. \\
\hline
\end{tabular}


Tabla 4. Usos/actividades identificadas en la zona marino - costera del departamento de Bolívar. Table 4. Uses/activities identified in the Bolivar Department marine and coastal area.

\begin{tabular}{|c|c|c|}
\hline $\begin{array}{l}\text { Actividades Marítimas } \\
\text { (Decreto Ley 2324/84) }\end{array}$ & Usos/ Actividades & Subdivisión de Uso/Actividad \\
\hline \multirow{2}{*}{ Control del tráfico maritimo } & Áreas de fondeo & Áreas de fondeo. \\
\hline & Canales de navegación & Canales de navegación. \\
\hline Administración y desarrollo de la zona costera & Concesiones Marítimas & $\begin{array}{l}\text { Acuicultura. } \\
\text { Industria. } \\
\text { Restaurantes. } \\
\text { Hoteles. } \\
\text { Marinas y Embarcaderos. } \\
\text { Emisarios Submarinos. }\end{array}$ \\
\hline Usos/ Actividades & \multicolumn{2}{|c|}{ Subdivisión de Uso/Actividad } \\
\hline Usos del suelo & $\begin{array}{l}\text { Agricultura. } \\
\text { Comercial. } \\
\text { Ganadería. } \\
\text { Industrias Camaroneras. } \\
\text { Institucional. } \\
\text { Lotes en zona urbana. } \\
\text { Protección de cuencas y } \\
\text { arroyos. }\end{array}$ & $\begin{array}{l}\text { Residencial. } \\
\text { Tierras sin uso. } \\
\text { Turismo. } \\
\text { Vegetación abierta sin uso. } \\
\text { Zona de reserva de defensa nacional. } \\
\text { Zona deforestada. } \\
\text { Zona de uso mixto }\end{array}$ \\
\hline
\end{tabular}

Aproximación AHP - Analytical Hierarchy Process para el establecimiento de los pesos para cada uso/ actividad

La aproximación AHP (Analytic Hierarchy Process: AHP) integra aspectos tanto cualitativos como cuantitativos en un proceso de decisión, permitiendo comparar pares de usos/actividades basados en la calificación de un elemento sobre otro a partir de niveles de importancia (Malczewski 1999; Arancibia et al., 2015; Osorio - Gómez \& Orejuela-Cabrera, 2008). Para OMC: VAM se utilizó la escala de intensidad de importancia de Saaty, 1980 (tabla 5).

Teniendo en cuenta la técnica de grupo nominal con juicio de expertos (Ouchi, 2004), se elaboraron las siguientes matrices:

1. Matriz de compensación de parejas. Se construyó teniendo en cuenta las relaciones de superposición $\mathrm{y}$ de conflicto entre los usos/actividades identificados en la zona marino - costera del departamento de Bo- lívar. Los diferentes usos/actividades se incluyeron tanto en las filas como en las columnas de la matriz, y se le asignaron valores de importancia $\left(V I_{i j}\right)$ con base a la opinión de los expertos y la ponderación de la escala de Saaty (tabla 5). De acuerdo con esto, la comparación de la importancia del $U_{s o_{i}}$ con respecto al Uso en la generación del posible conflicto (tabla 6), se basa en los valores de la escala de Saaty y sus recíprocos en una matriz simétrica $\left(V I_{i j}=1 / V I_{j i}\right)$ (Villa et al., 2002; DIMAR, 2019a, Afanador-Franco et al., 2019).

Los valores se asignan por filas teniendo en cuenta que existe una relación directa entre importancia y conflicto. En la tabla 6, por ejemplo, la calificación del Uso1 con respecto al Uso2 indica que el Uso1 contribuye menos en el conflicto con el Uso2, y su recíproco, muestra que el Uso2 contribuye de forma fuerte en el conflicto con el Uso1. 


\section{rig COSTAS}

\begin{tabular}{|c|l|}
\hline \multicolumn{2}{|c|}{$\begin{array}{c}\text { Tabla 5. Escala de Saaty (Malczewski, 1999). } \\
\text { Table 5. Saaty Scale (Malczewski, 1999). }\end{array}$} \\
\hline Intensidad de la Importancia & \multicolumn{1}{c|}{ Definición } \\
\hline $\mathbf{1}$ & Igual importancia \\
\hline $\mathbf{2}$ & Igual a moderada importancia \\
\hline $\mathbf{3}$ & Moderada importancia \\
\hline $\mathbf{4}$ & Moderada a fuerte importancia \\
\hline $\mathbf{5}$ & Fuerte importancia \\
\hline $\mathbf{6}$ & Fuerte a muy fuerte importancia \\
\hline 7 & Muy fuerte importancia \\
\hline $\mathbf{8}$ & Muy a extremadamente fuerte importancia \\
\hline $\mathbf{9}$ & Extrema importancia \\
\hline
\end{tabular}

Tabla 6. Ejemplo de matriz de compensación por parejas para 4 usos (DIMAR 2019a).

Table 6. Example of pairwise compensation matrix for 4 uses (DIMAR 2019a).

\begin{tabular}{|c|c|c|c|c|}
\hline Uso/Actividad & Uso 1 & Uso 2 & Uso 3 & Uso 4 \\
\hline Uso 1 & 0 & $1 / 5$ & $1 / 6$ & 4 \\
\hline Uso 2 & 5 & 0 & $1 / 3$ & 4 \\
\hline Uso 3 & 6 & 3 & 0 & 6 \\
\hline Uso 4 & $1 / 4$ & $1 / 4$ & $1 / 6$ & 0 \\
\hline$\Sigma$ & 11.250000 & 3.450000 & 0.670000 & 14.000000 \\
\hline
\end{tabular}

2. Matriz de justificación: de forma simultánea a la asignación de pesos, se estructuró una matriz de justificación en donde se incluyó de manera breve la razón de la asignación de los valores de importancia en la matriz de compensación por parejas (DIMAR, 2019a).

3. Matriz mormalizada de compensación por parejas: para elaborar esta matriz, se dividió cada uno de los valores de las celdas de la matriz de compensación por parejas entre la suma de su respectiva columna, lo que permitió obtener valores de conflicto entre 0 y 1 (DIMAR 2019a; Afanador-Franco et al., 2019).

$$
\begin{aligned}
V I N_{i j} & =V I_{i j} / \sum_{k=}^{p} \\
\text { Para } 1 & \leq i \leq p \\
1 & \leq j \leq q
\end{aligned}
$$

Donde $V I N_{i j}$ es el valor de importancia normalizado. $V I_{i j}$ Es el valor de importancia entre cada par de usos/actividades. $p$ Número de filas de la matriz. $q$ Número de columnas de la matriz (tabla 7).

4. Matriz de pesos: el cálculo de los pesos de cada uso/actividad se llevó a cabo sumando, para cada fila de la matriz normalizada, los valores de las celdas y el resultado se dividió entre el número de usos/actividades identificados para la zona de trabajo (DIMAR, 2019a; Afanador-Franco et al., 2019).

$$
\text { Wur }_{i}=\left(\sum_{k=1}^{q} \operatorname{VIN}_{i k}\right) / p
$$


Tabla 7. Ejemplo de matriz normalizada de compensación por parejas (DIMAR 2019a. Table 7. Example of normalized pairwise compensation matrix (DIMAR 2019a).

\begin{tabular}{|c|c|c|c|c|}
\hline Uso/Actividad & Uso 1 & Uso 2 & Uso 3 & Uso 4 \\
\hline Uso 1 & 0.000000 & 0.057971 & 0.250000 & 0.285714 \\
\hline Uso 2 & 0.444444 & 0.000000 & 0.500000 & 0.285714 \\
\hline Uso 3 & 0.533333 & 0.869565 & 0.000000 & 0.428571 \\
\hline Uso 4 & 0.022222 & 0.072464 & 0.250000 & 0.000000 \\
\hline $\boldsymbol{\Sigma}$ & 1.000000 & 1.000000 & 1.000000 & 1.000000 \\
\hline
\end{tabular}

Tabla 8. Ejemplo de matriz normalizada de comparación por parejas con el cálculo de los respectivos pesos (DIMAR 2019a).

Table 8. Example of normalized pairwise compensation matrix calculating weight (DIMAR 2019a).

\begin{tabular}{|c|c|c|c|c|c|}
\hline Uso/Actividad & Uso 1 & Uso 2 & Uso 3 & Uso 4 & Pesos \\
\hline Uso 1 & 0.000000 & 0.057971 & 0.250000 & 0.285714 & 0.148421 \\
\hline Uso 2 & 0.444444 & 0.000000 & 0.500000 & 0.285714 & 0.307540 \\
\hline Uso 3 & 0.533333 & 0.869565 & 0.000000 & 0.428571 & 0.457867 \\
\hline Uso 4 & 0.022222 & 0.072464 & 0.250000 & 0.000000 & 0.086171 \\
\hline $\boldsymbol{\Sigma}$ & 1.000000 & 1.000000 & 1.000000 & 1.000000 & 1.000000 \\
\hline
\end{tabular}

Donde, $W_{u} r_{i}$ es el peso relativo del Uso ${ }_{i}$ basado en juicio de expertos; $\sum_{k=1}^{q} V I N_{i k}$ es la sumatoria de los valores de importancia normalizados $p$ número de filas de la matriz (tabla 8).

El resultado de las matrices mostró que el Área Marina Protegida de los Archipiélagos del Rosario y de San Bernardo (AMP-ARSB) $\left(W_{u r}=0.11\right)$, los cables submarinos $\left(W_{u r}=0.10\right)$, las actividades turísticas $\left(W_{u r}=0.09\right)$, El Parque Nacional Natural Corales del Rosario y de San Bernardo (PNN-CRSB) ( $W_{u r}$ $=0.08)$ y las zonas destinadas para concesiones portuarias $\left(W_{u r}=0.07\right)$, son los usos/actividades que más peso tienen y por lo tanto son los que más contribuyen al conflicto con respecto a los demás en el área de estudio (DIMAR, 2019b; Afanador-Franco et al., 2019).

\section{Cuantificación de indice de conflicto} por pareja de usos/actividades

Para calcular el índice de conflicto, se analizaron los usos/actividades que presentaron superposiciones y conflictos empleando herramientas SIG. Se incluyó en cada capa temática de uso/actividad, el valor del peso calculado en la matriz normalizada, luego se realizaron operaciones de intersección entre los usos y se sumaron ambos pesos (Malczewski, 1999; Tuda et al., 2014).

$$
I C=W u r_{i}+W u r_{j} 3
$$

Donde, IC es el índice de conflicto; Wur es el peso del usoi y Wur es el peso del uso $j$. 


\section{Fin COSTAS}

Finalmente, para obtener valores estandarizados de índice de conflicto entre 0 y 1 se integraron todos los conflictos de cada uso, y se calculó el índice de conflicto normalizado utilizando la siguiente expresión:

$$
I C N=\left(I C_{i}-I C_{i \text { mín }}\right) /\left(I C_{i \text { máx }}-I C_{i \text { mín }}\right)^{4}
$$

Donde, ICN es el índice de conflicto normalizado, $I C_{i}$ corresponde al índice de conflicto de cada par de usos en la capa de conflictos que se esté analizando, $I C_{i \text { min }}$ corresponde al valor más bajo de índice de conflicto en la capa de conflictos que se esté analizando e $I C_{i \max }$ corresponde al valor más alto de índice de conflicto en la capa de conflictos que se esté analizando (DIMAR 2019b; Afanador-Franco et al., 2019). Los valores obtenidos se categorizaron de acuerdo a la escala presentada en la Tabla 9.

Teniendo en cuenta los niveles de clasificación de índices de conflicto normalizado se elaboró el mapa de zonificación por índice de conflicto (figura 4).

\section{Superposición de conflictos entre usos/actividades}

Considerando la cantidad de conflictos superpuestos en el área de estudio se realizó una Zonificación por número de conflictos basados en un rango de valores establecidos a partir del juicio de expertos (tabla 10; figura 5).

Como resultado de este proceso se identificó que los cables submarinos, los sitios de conservación de la plataforma, el AMP-ARSB, los bajamares, las actividades turísticas y las playas, son los usos/actividades que presentaron el mayor número de traslapes y conflictos con respecto a los demás en la zona marino - costera del departamento de Bolívar (DIMAR, 2019b; Afanador-Franco et al., 2019).

\section{Identificación de zonas libres de conflicto}

Estas zonas representan los espacios libres identificados y delimitados a partir de la zonificación por índice y número de conflictos por medio de un proceso SIG, en el cual se extraen las áreas ocupadas por los diferentes usos/actividades, y corresponden a áreas en donde se podrían desarrollar nuevos usos/actividades considerando sus aspectos técnicos, operacionales y normativos (Douvere \& Ehler, 2009), de tal forma que se garantice un funcionamiento óptimo de cada uno de ellos. En total se cuantificaron $43786.68 \mathrm{~km}^{2}$ de zonas libres de conflictos, distribuidos entre la zona A y B del área de estudio (figura 6).

\section{Análisis de condiciones futuras}

La metodología OMC: VAM considera que el crecimiento y desarrollo de las actividades marítimas a nivel mundial es constante, multidimensional (Douvere, 2010; Bonnevie et al., 2019; Ansong et al., 2019) y compuesto de múltiples interrelaciones (Ehler et al., 2019), por lo que es importante analizar diferentes escenarios de gestión, criterios ambientales, técnicos y socioeconómicos para poder asignar el espacio disponible de manera óptima (Lester et al., 2013; Coccoli et al., 2018).

A partir de lo anterior, se elaboró empleando herramientas SIG, una metodología para el análisis de

Tabla 9. Niveles de clasificación de índices de conflicto normalizado (DIMAR, 2019a). Table 9. Classification levels of a normalized conflict index (DIMAR, 2019a).

\begin{tabular}{|c|c|}
\hline Categoría & Rango ICN \\
\hline Alto & $0.5-1$ \\
\hline Medio & $0.250-0.499$ \\
\hline Bajo & $0.000-0.249$ \\
\hline
\end{tabular}


Afanador Franco et al.

\section{reg \\ COSTAS}

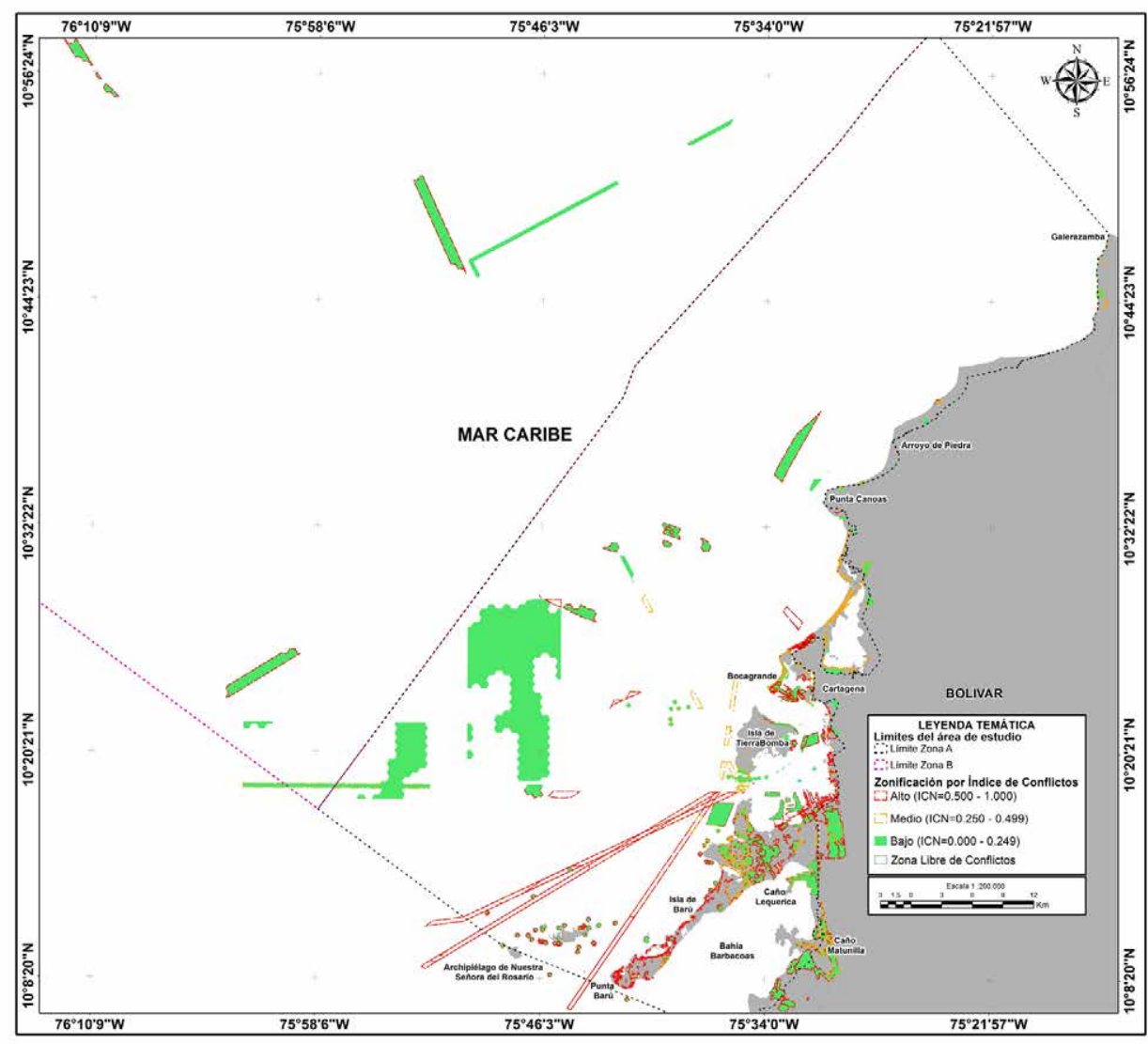

Figura 4. Zonificación por índice de conflictos en la zona marino - costera del departamento de Bolívar (DIMAR, 2019b).

Figure 4. Zoning by the index of conflict in Bolivar Department marine and coastal areas (DIMAR, 2019b).

optimización de la localización espacial de usos/actividades a partir del Modelo de Asignación y Co-localización - MAYC, la cual establece tres escenarios que se pueden presentar ante la Autoridad Marítima: localización espacial preestablecida, corresponde a un escenario en el cual el usuario solicita un espacio para un uso/actividad, y hace entrega de las coordenadas geográficas; localización espacial no conoci$d a$, parte del supuesto de que un usuario cuenta con la información relacionada de las características generales del uso/actividad pero no ha definido su ubicación espacial; análisis en el área actual, plantea que un usuario dispone de la información relacionada con su uso/actividad (coordenadas geográficas) de tal forma que al momento de ubicarlo espacialmente se encuentre en un espacio donde ya se desarrollan otros usos/actividades (DIMAR, 2019a; Afanador-Franco et al., 2019) (figura 7).

\section{Análisis de asignación}

Para su ejecución se tienen en cuenta las zonas libres de usos y los criterios técnicos establecidos para cada uno de los usos/actividades (Afanador-Franco et al., 2019):

Sea $U_{1}, U_{2}, U_{3}, \ldots, U_{n}$ la totalidad de los usos relacionados con actividades marítimas 


\section{Fin \\ COSTAS}

Tabla 10. Niveles de clasificación por número de conflictos (DIMAR 2019a).

Table 10. Classification levels by the number of conflicts (DIMAR 2019a).

\begin{tabular}{|c|c|}
\hline Categoría & No. Conflictos \\
\hline Alto & $>6$ \\
\hline Medio & $4-6$ \\
\hline Bajo & $1-3$ \\
\hline
\end{tabular}

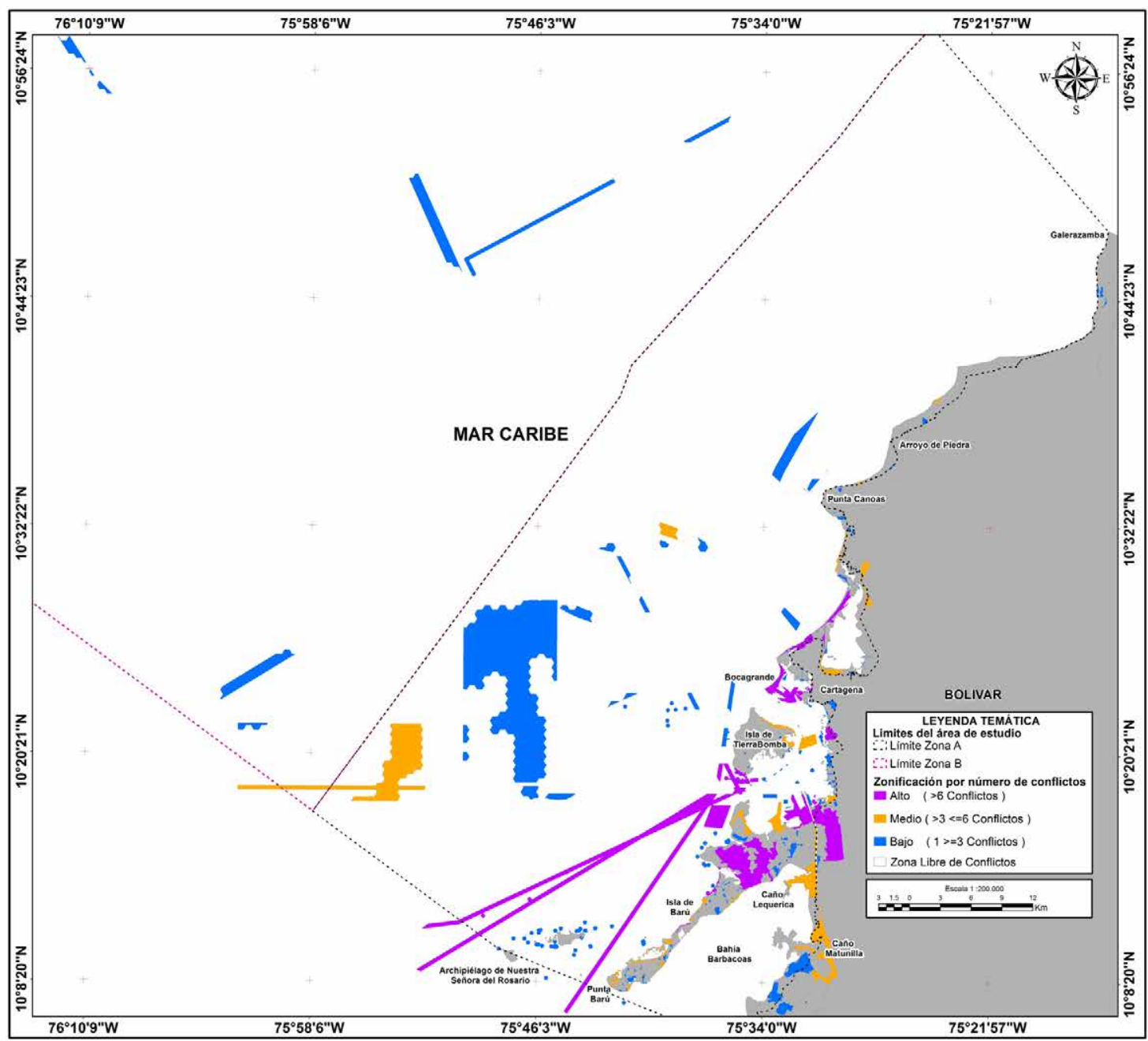

Figura 5. Zonificación por número de conflictos en la zona marino - costera del departamento de Bolívar (DIMAR, 2019b).

Figure 5. Zoning by number of conflicts in Bolivar Department marine and coastal area (DIMAR, 2019b). 


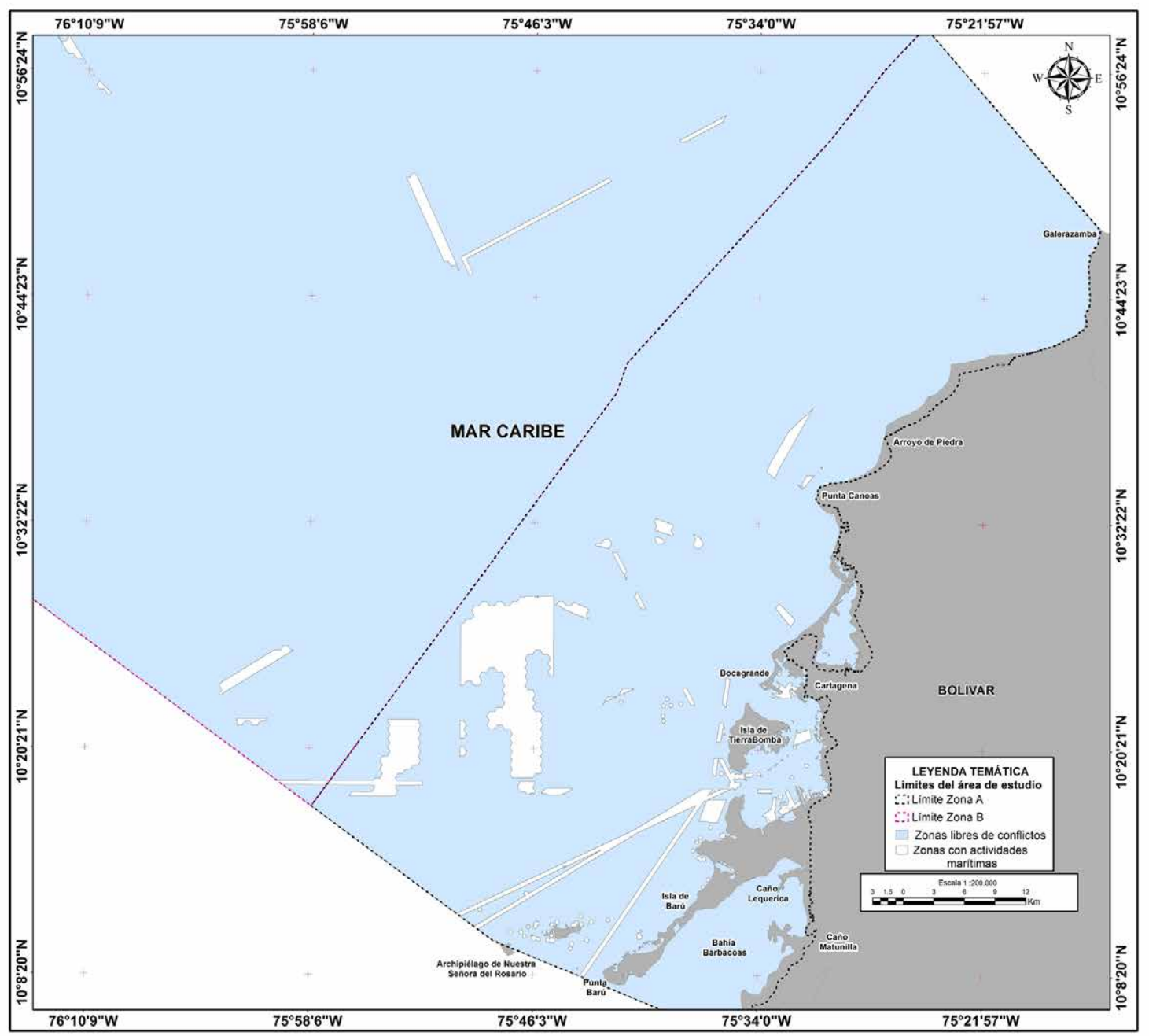

Figura 6. Áreas libres de conflictos en la zona marino - costera del departamento de Bolívar (DIMAR, 2019b).

Figure 6. Free areas of Bolivar Department marine and coastal area (DIMAR, 2019b).

Sea $U_{x}$ el uso/actividad para la cual se requiere conocer su localización óptima

Para $U_{x}$, se define:

Crit $_{1}$, Crit $_{2}$, Crit $_{3}, \ldots$, Crit $_{n}$ que corresponden a los criterios para determinar la localización más óptima $\operatorname{del} U_{x}$.

Sea:

$$
\text { WCrit }_{i}=W_{i} \times \text { Crit }_{i} \quad 1
$$

Donde $W C$ rit ${ }_{i}$ corresponde al peso del criterio $i$, establecido con base en la revisión del estado del arte disponible sobre criterios para la localización óptima de usos/actividades.

Para un $U_{x}$ :

$\sum_{i=1}^{n}$ Writ $_{i}=1 \times 100 \% \quad i=1,2,3, \ldots, \eta \quad 2$ 


\section{rin \\ COSTAS}

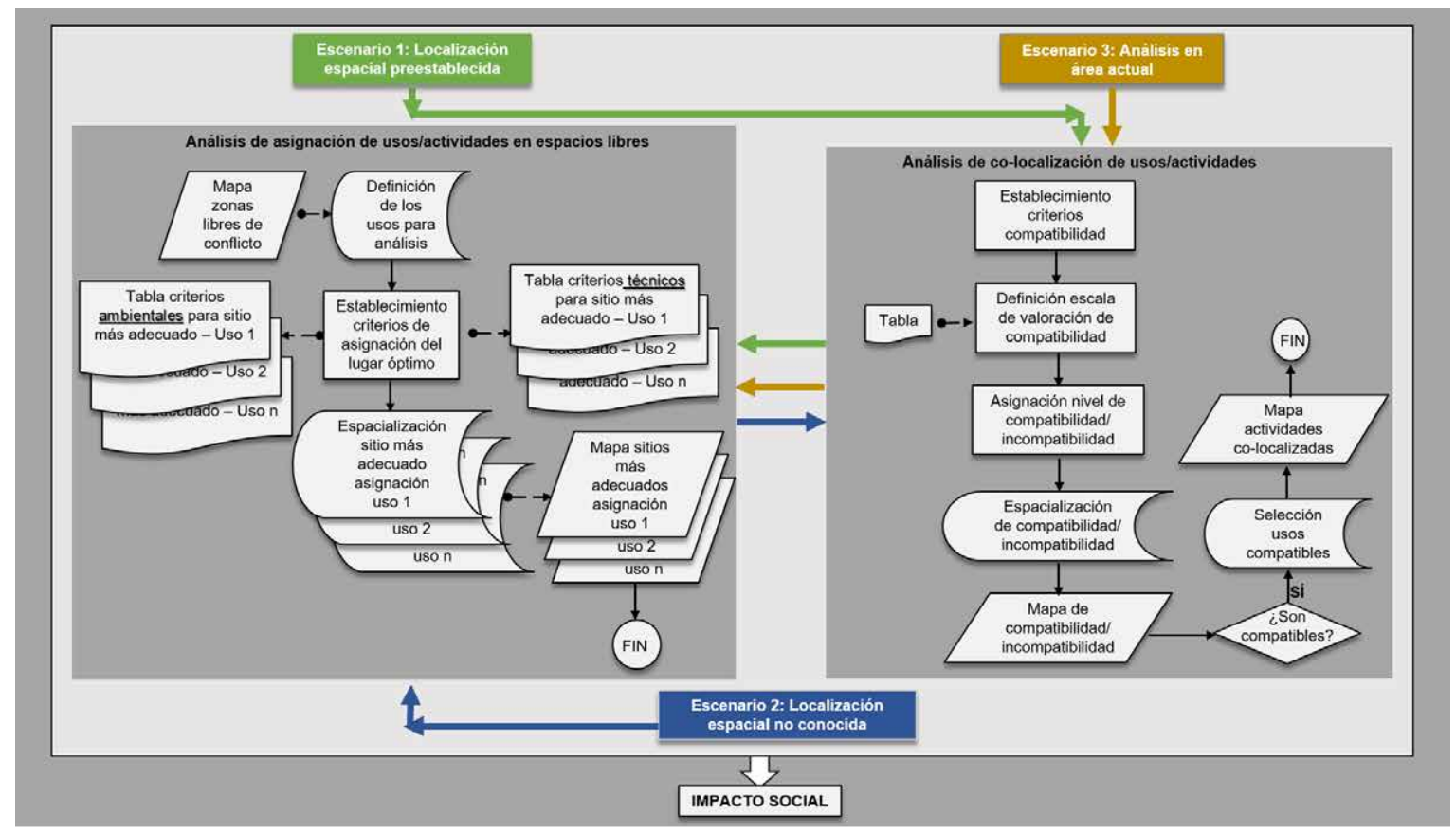

Figura 7. Etapas del Modelo de Asignación y Co-localización - MAYC para la localización de nuevos usos/actividades en las zonas marino - costeras (DIMAR, 2019a).

Figure 7. Allocation and Co-location Model (ACM) stages to locate new uses/activities in marine and coastal areas (DIMAR, 2019a).

Donde WCrit cercano a 100 es el criterio más importante para la localización del $U_{s o}$, según lo establecido por el juicio de expertos.

Para todo Crit existen: $^{2}$

SCrit, SCrit ${ }_{2}, S$ Crit $_{3}, \ldots, S$ Crit ${ }_{p}$ que corresponden a los subcriterios (atributos) del criterio

Sea:

$$
\text { QSCrit }_{k}=Q_{k} \times \text { SCrit }_{k} \quad 3
$$

Donde QSCrit, corresponde al peso del subcriterio $k$

Para un Crit:

$\sum_{k=1}^{p}$ QSCrit $_{k}=1 \quad k=1,2,3, \ldots, p \quad 4$

A partir del juicio de expertos, se clasifican los subcriterios en óptimos, medianamente óptimos y no

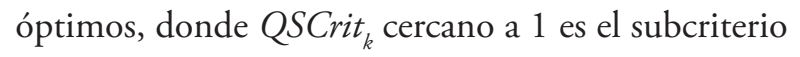
más óptimo dentro Crit. 


\section{Discusión}

Según la IOC-UNESCO (2006-2020a), alrededor de 70 países cuentan con iniciativas de Planificación Espacial Marina (PEM), que en general buscan una armonía entre el desarrollo de las actividades marítimas y el ecosistema, a través del establecimiento de mecanismos de resolución de conflictos y la integración de diferentes perspectivas con los actores involucrados. Estos procesos utilizan herramientas SIG, análisis multicriterio y técnicas de optimización, para identificar los conflictos de uso en un área determinada, sin embargo no existe un único proceso metodológico, ya que su desarrollo e implementación pueden variar dependiendo de la geografía, presiones marinas, requisitos legales, culturas, entre otras, propias de cada país (Vallega, 1999; Ehler, 2008; Ehler \& Douvere ,2009; Leslie \& McLeod, 2007; Regan et al., 2007; Jay, 2017).

En 2009 la Comisión Oceanográfica Intergubernamental (COI) de la UNESCO como resultado de varios talleres sobre el uso de la planificación espacial marina, elaboró la primera guía internacional de PEM "Un enfoque paso a paso", la cual presenta un proceso sistemático que abarca diferentes etapas para el análisis de condiciones actuales y futuras, y tiene la ventaja de articularse con otros procesos de ordenamiento y gestión de zonas marino - costeras, razón por la cual se convirtió en un modelo reconocido internacionalmente (Ehler \& Douvere, 2009; Jay, 2017; ICO-UNESCO, 2006-2020b).

Australia, China, Norteamérica, Canadá, Alemania entre otros países, han implementado la PEM, analizando un modelo sectorial específico de actividades como pesca, puertos, minería, turismo, Áreas Marinas Protegidas, lo cual limita el número de usos y actores involucrados, facilitando la coordinación y la resolución de conflictos (Jones et al., 2016; Leslie \& McLeod 2007; Lester et al., 2013; Tuda et al., 2014).
En Colombia, diferentes entidades gubernamentales, han llevado a cabo ejercicios de PEM, mediante la aproximación cualitativa propuesta por la COI-UNESCO donde se valoran compatibilidades e incompatibilidades entre las diferentes actividades marino costeras (Ehler \& Douvere, 2009; INVEMAR, 2017). Sin embargo, la DIMAR teniendo en cuenta su naturaleza jurídica como Autoridad Marítima, propone una metodología que aunque presenta una sinergia con los modelos internacionales y nacionales de PEM, incluye un enfoque cuantitativo basado en procesos AHP, que involucra el análisis de diferentes sectores entre los cuales se pueden generar conflictos que afectan el desarrollo de los procesos de ordenamiento en los espacios marino - costeros (Malczewski, 1999; Leslie \& McLeod, 2007; Lester et al., 2013; Tuda et al., 2014; Jones et al., 2016; DIMAR, 2019a).

Teniendo en cuenta lo anterior, esta metodología permitió identificar que los cables submarinos, el AMP-ARSB y el turismo, son los tres usos que en el departamento de Bolívar, ocupan el mayor espacio geográfico y por sus características técnicas y legales, tienen un nivel de conflicto superior con respecto a las demás actividades marítimas analizadas (Afanador-Franco et al., 2019).

Los cables submarinos transportan aproximadamente el $90 \%$ de datos y comunicaciones a nivel mundial, razón por la cual son considerados importantes para la economía y seguridad de todos los países del mundo, y deben tener un alto nivel de protección ya que por actividades como la pesca, la minería, el anclaje de embarcaciones, entre otras, pueden sufrir daños (Zevallos-León, 2008; Carter et al., 2009; Naciones Unidas, 2011). Teniendo en cuenta lo anterior la DIMAR estipulo en la Resolución 204 de 2012 una zona de seguridad de $500 \mathrm{~m}$ a cada lado del 


\section{re COSTAS}

tendido de cables en aguas jurisdiccionales (DIMAR, 2012).

El AMP-ARSB creada mediante la Resolución 0679 de 2005 del Ministerio de Ambiente, Vivienda y Desarrollo Territorial, busca reducir los efectos de las actividades que se desarrollan en las zonas marino costeras sobre los ecosistemas, estableciendo medidas para la conservación del medio natural a través de la definición de siete objetivos (MinAmbiente et al. 2016), los cuales se tuvieron en cuenta para realizar el análisis del AMP-ARSB en comparación con cada uno de los demás usos (Afanador-Franco et al., 2019).

La capital del departamento de Bolívar debido a sus características históricas, culturales, naturales entre otras, presenta una alta actividad turística (UNESCO, 2014), que se ha desarrollado de forma desordenada a lo largo del litoral, sin embargo es justificada por los altos beneficios económicos que esta

\section{Conclusiones}

La metodología para el OMC: VAM, contribuye al desarrollo de la Planificación Espacial Marina a nivel mundial, como una herramienta para el fortalecimiento de la economía marítima, la conservación de la base ecosistémica y la cooperación transfronteriza (Schultz-Zehden et al., 2019). Asimismo, es un punto de inicio para el ordenamiento marino costero de Colombia, ya que integra el análisis de condiciones actuales y futuras teniendo en cuenta los intereses marítimos nacionales y el concepto de gobernanza, aportando a la generación de instrumentos para la gestión y manejo de los espacios marinos, costeros e insulares del país.

En la zona marino-costera del departamento de Bolívar se identificaron 55 usos, localizados principalmente en la Bahía interna de Cartagena de Indias, entre los cuales se analizaron compatibilidades e in-
Revista Costas, Vol. Esp. 2, 2021

actividad genera (Ministerio del Medio Ambiente, 2001; Papageorgiou, 2016).

Finalmente, ante el crecimiento de diferentes sectores económicos y sociales, la Autoridad Marítima desde el OMC:VAM, plantea una metodología de Asignación y Co-Localización en la cual se busca analizar la mejor ubicación de usos con tendencia al desarrollo como, la actividad turística, la acuicultura, el tráfico marítimo, entre otros, a partir de la identificación de lugares en los cuales se cumplan ciertas características técnicas y ambientales que permitan un desarrollo óptimo de cada uno de ellos, y además plantea escenarios futuros que pretenden identificar oportunidades, conflictos y compatibilidades, para orientar la toma de decisiones entendiendo las implicaciones de las actividades marítimas futuras (Schultz-Zehden et al., 2019; Ehler \& Douvere, 2009; Afanador-Franco et al., 2019).

compatibilidades, estableciendo áreas con niveles altos, medios y bajos de conflictos dependiendo de los pesos asignados, obteniendo así que el Área Marina Protegida, cables submarinos, turismo, Parques $\mathrm{Na}$ cionales Naturales y concesiones portuarias presentan el mayor índice de conflicto.

Así mismo se identificaron zonas en donde convergen hasta 12 superposiciones de conflictos por uso, los cuales fueron representados por medio de un mapa de Zonificación por número de conflictos. Los seis usos con más conflictos debido a sus características de desarrollo y ocupación geográfica, fueron cables submarinos, sitios de conservación de la plataforma, Área Marina Protegida, bajamares, turismo y playas.

A partir del análisis de la información anterior y el uso de herramientas SIG, se definieron y calcularon 
las áreas libres de usos y libres de conflictos, las cuales representan zonas en las que se podrían desarrollar futuros usos/actividades y son la base para la apli- cación del modelo de Asignación y Co-localización (MAYC) en la zona marino - costera del departamento de Bolívar.

\section{Agradecimientos}

Los autores expresan sus agradecimientos a la Dirección General Marítima por el apoyo y financiación de la presente investigación enmarcada dentro del proyecto "Planificación y Ordenamiento de los litorales y áreas marinas Colombianas".

\section{Referencias}

Afanador-Franco F, Molina-Jiménez MP, Pusquin-Ospina LT, Escobar-Olaya G, Castro-Mercado I. 2019. Conflictos de Uso en el Proceso de Ordenamiento Marino Costero: Visión de Autoridad Marítima. Departamento de Bolívar - Colombia. Boletín Científico CIOH. 38(1): 27-40.

Agencia de inversiones de Cartagena de Indias y Bolívar. Unpublished 2012. Cartagena y Bolívar: una ubicación estratégica para el comercio exterior.

Aldana O, Hernández A. 2016. La Planificación Espacial Marina: marco operativo para conservar la diversidad biológica marina y promover el uso sostenible del potencial económico de los recursos marinos. In: Hernández-Zanuy A. C, editor. Adaptación basada en Ecosistemas: alternativa para la gestión sostenible de los recursos marinos y costeros del Caribe. La Habana: Editorial Instituto de Oceanología. p.109 - 122.

Alvarado E, Pizarro V, Sarmiento-Segura A. 2011. Formaciones Arrecifales. In: Zarza E, editor. El entorno ambiental del Parque Nacional Natural Corales del Rosario y San Bernardo. 1th ed. Colombia: Quito Publicidad. pp. 109 - 123.

Andrade-Amaya C. 2001. Las corrientes superficiales en la Cuenca de Colombia observadas con boyas de deriva. Revista de la Academia Colombiana de Ciencias Exactas, Físicas y Naturale, 25 (96): 321-335.

Ansong J, Calado H, Gilliland P. M. 2019. A multifaceted approach to building capacity for marine/maritime spatial planning based on European experience. $\mathrm{Ma}$ rine Policy [Submitted].

Arancibia S, De la Vega L, Denis A, Saball P. 2015. Evaluación de programas sociales: un enfoque multicriterio. Revista del CLAD Reforma y Democracia, 63: 99-126.

Audemard F, Audemard F. 2002. Structure of the Merida Andes, Venezuela: relations with the South America-Caribbean geodynamic interaction. Tectonophysics, 345: 299-327.

Bonnevie I, Hansen H, Schrøder L. 2019. Assessing useuse interactions at sea: A theoretical framework for spatial decision support tools facilitating co-location in maritime spatial planning. Marine Policy, 106: 103533.

Brown K, Adger W N, Tompkins E, Bacon P, Shim D, Young K. 2001. Trade-off analysis for marine protected area management. Ecological Economics, 37: 417434.

Bueger C. 2015. What is maritime security?. Marine Policy, 53: 159-164.

[CARDIQUE] Corporación Autónoma Regional del Canal del Dique. Unpublished 1998. Diagnóstico, zonificación y planificación estratégica de las áreas de manglar de Bolívar. Cartagena (Colombia).

Carter L, Burnett D, Drew S, Marle G, Hagadorn L, Bartlett-McNeil D, Irvine N. 2009. Submarine Cables and the Oceans - Connecting the World. Cambridge (United Kingdom): ICPC/UNEP/UNEP-WCMC.

Carvajal A, Jurado J. 2009. Caracterización físico-biótica del litoral del departamento de Bolívar. In: DIMAR ed. Caracterización físico-biótica del litoral Caribe 


\section{rig \\ COSTAS}

colombiano. Cartagena (Colombia): Editorial DIMAR. 2nd Vol. p. 13 - 38.

[CCO] Comisión Colombiana del Océano. 2018. Política Nacional del Océano y de los Espacios Costeros - PNOEC. Bogotá (Colombia): Secretaría Ejecutiva CCO.

[CEDEC] Centro de Estudios para el Desarrollo y la Competitividad, [CCC] Cámara de Comercio de Cartagena. Unpublished 2018. Coyuntura Económica de Cartagena mayo 2018.

Christie N, Smyth K, Barnes R, Elliott M. 2014. Co-location of activities and designations: A means of solving or creating problems in marine spatial planning?. $\mathrm{Ma}$ rine Policy, 43: 254-261.

Cicin-Sain B, Knecht R. 1998. Integrated coastal and ocean management: concepts and practices. Washington, D.C., Unites States of America: Island press.

Coccoli C, Galparsoro I, Murillas A, Pınarbaş K, Fernandes J. 2018. Conflict analysis and reallocation opportunities in the framework of marine spatial planning: A novel, spatially explicit Bayesian belief network approach for artisanal fishing and aquaculture. Marine Policy, 94: 119-131.

De Groot R, Wilson M, Boumans R. 2002. A typology for the classification, description and valuation of ecosystem functions, goods and services. Ecological Economics, $41: 393-408$.

Díaz J, Barrios L, Cendales M, Garzón-Ferreira J, Geister J, López-Victoria M, Ospina G, Parra-Velandia F, Pinzón J, Vargas-Ángel B, et al. 2000. Áreas coralinas de Colombia. Santa Marta (Colombia): INVEMAR Serie Publicaciones Especiales No. 5.

[DIMAR] Dirección General Marítima. 2012. Resolución $\mathrm{N}^{\circ} 20419$ de abril de 2012. Bogotá (Colombia): Diario Oficial 48.410.

[DIMAR] Dirección General Marítima. 2018. Plan Estratégico de Desarrollo 2030. Bogotá (Colombia): Área de comunicaciones estratégicas DIMAR.

[DIMAR] Dirección General Marítima. Unpublished 2019a. Lineamientos Técnicos para el Ordenamiento Marino Costero: Visión de Autoridad Marítima OMC: VAM. Cartagena (Colombia).

[DIMAR] Dirección General Marítima. Unpublished 2019b. Ordenamiento Marino Costero (OMC): Visión De Autoridad Marítima. Departamento de Bolívar. Sector Galerazamba - Caño Matunilla. Cartagena D.T. y C., Colombia.
Revista Costas, Vol. Esp. 2, 2021

[DNP] Departamento Nacional de Planeación. 2019. Bases del Plan Nacional de Desarrollo 2018-2022: Pacto por Colombia, pacto por la equidad. Bogotá (Colombia): Grupo de Comunicaciones Villamizar C, Patiño G, Bayona C, Bonilla C.

[DNP] Departamento Nacional de Planeación. 2020. Documento Consejo Nacional de Política Económica y Social CONPES 3990. Bogotá (Colombia).

Douvere F, Ehler C. 2009. New perspectives on sea use management: initial findings from European experience with marine spatial planning. Journal of Environmental Management, 90: 77-88.

Douvere F, Maes F, Vanhulle A, Schrijvers J. 2007. The role of marine spatial planning in sea use management: the Belgian case. Marine Policy, 31: 182-191.

Douvere F. 2010. Marine spatial planning: concepts, current practice and linkages to other management approaches [dissertation]. [Belgium]: Ghent University.

Ehler C, Douvere F. 2009. Marine Spatial Planning: a step-by-step approach toward ecosystem-based management. Paris (France): UNESCO IOC.

Ehler C, Zaucha J, Gee K. 2019. Maritime/marine spatial planning at the interface of research and practice. In: Zaucha J, Gee K, editors. Maritime Spatial Planning past, present, future. Cham (Switzerland): Springer International Publishing AG. p. 1 - 21.

Ehler C. 2008. Conclusions: Benefits, lessons learned, and future challenges of marine spatial planning. Marine Policy, 32: $840-843$.

[EP\&C] European Parliament and of the Council. 2013. Establishing a Framework for Maritime Spatial Planning and Integrated Coastal Management. Brussels.

European Commission. 2019. The EU Blue Economy Report 2019. Luxembourg: Publications Office of the European Union.

European Commission. 2020. The EU Blue Economy Report 2020. Luxembourg: Publications Office of the European Union.

Fang Q, Zhu S, Ma D, Zhang L, Yang S. 2019. How effective is a marine spatial plan: An evaluation case study in China. Ecological Indicators, 98: 508-514.

[FAO] Organización de las Naciones Unidas para la Alimentación y la Agricultura. 2014. El estado mundial de la pesca y la acuicultura Oportunidades y desafíos. Roma.

Farahani R, Hekmatfar M, editors. 2009. Facility location: concepts, models, algorithms and case studies. 
Berlin - Heiderlberg (Gemany): Springer Science \& Business Media. Springer.

Franco Herrera A, Sánchez Muñoz J. A, Guerra Curvelo W, Reynolds Pombo J, Speich S, Cantera Kintz, J, Gutierrez Bonilla F, Peńa Salamanca, E. 2020. Colombia, la oportunidad del agua: dos océanos y un mar de ríos y aguas subterráneas. 1st ed. Bogotá (Colombia): Editorial UTadeo. 7 Vol.

Frazão C, Ehler C, Agardy T, Andrade F, Orbach M, Crowder L. 2019. Marine Spatial Planning. In: Sheppard C, editor. World Seas: An Environmental Evaluation. 2nd ed. London (United Kingdom): Academic Press. 3 vol. p. 571-592.

Gilliland P, Laffoley D. 2008. Key elements and steps in the process of developing ecosystem-based marine spatial planning. Marine Policy, 32: 787-796.

Gopnik M, Fieseler C, Cantral L, McClellan K, Pendleton L, Crowder L. 2012. Coming to the table: Early stakeholder engagement in marine spatial planning. Marine Policy, 36: 1139-1149.

Hennessey T.M, Sutinen J.G, editors. 2005. Large Marine Ecosystem. Narragansett (Rhode Island): Elsevier B.V. 13 Vol.

[IDEAM]. Instituto de Hidrología, Meteorología y Estudios Ambientales, [IGAC]. Instituto Geográfico Agustín Codazzi, [IAvH] Instituto de Investigación de Recursos Biológicos Alexander von Humbold, [Invemar] Instituto de Investigaciones Marinas y Costeras José Benito Vives De Andréis, [I. Sinchi] Instituto Amazónico de Investigaciones Científicas Sinchi, [IIAP] Instituto de Investigaciones Ambientales del Pacífico Jhon von Neumann. 2017. Ecosistemas Continentales, Costeros y Marinos de Colombia. Bogotá (Colombia): Imprenta Nacional de Colombia.

[INGEOMINAS] Instituto de Investigación e Información Geocientífica, Minero-Ambiental y Nuclear. 2001. GEOLOGÍA DE LAS PLANCHAS 23 CARTAGENA Y 29 - 30 ARJONA - Memoria Explicativa. Bogotá (Colombia): INGEOMINAS.

Inniss L, Simcock A, Yoanes A, Alcala A, Bernal P, Calumpong H, Araghi P, Green S, Harris P, Keh-Kamara O, et al. Unpublished 2016. The First Global Integrated arine Assessment World Ocean Assessment I. United Nations.

[INVEMAR] Instituto de Investigaciones Marinas y Costeras José Benito Vives De Andréis. 2017. Documento técnico de investigación: Planificación espacial marina para la zona costera del Cauca. Santa Marta (Colombia).

IOC-UNESCO: MSP Around the Globe (2006 - 2020a). Paris (France): Intergovernmental Oceanographic Commission and United Nations Educational, Scientific and Cultural Organization; [accessed 2020 oct 10]. http://msp.ioc-unesco.org/world-applications/ overview/.

IOC-UNESCO: MSP at ICO-UNESCO. (2006 2020b). Paris (France): Intergovernmental Oceanographic Commission and United Nations Educational, Scientific and Cultural Organization; [accessed 2020 oct 10]. http://msp.ioc-unesco.org/about/ msp-at-unesco/.

Jay S. 2017. Marine Spatial Planning: Assessing net benefits and improving effectiveness. Liverpool (United Kingdom): OECD Publishing.

Jones P. J, Lieberknecht L. M, Qiu W. 2016. Marine spatial planning in reality: Introduction to case studies and discussion of findings. Marine Policy,71: 256264.

Kennedy M, David-Ford E, Singleton P, Finney M, Agee J. K. 2008. Informed multi-objective decision-making in environmental management using Pareto optimality. Journal of Applied Ecology, 45: 181-192.

Leblé S, Cuignon R. 1987. El Archipiélago de las Islas del Rosario, Estudio morfológico, hidrodinámico y sedimentológico. Boletin Cientifico CIOH, 7: 37-52.

Leslie H. M, McLeod K. L. 2007. Confronting the challenges of implementing marine ecosystem-based management. Frontiers in Ecology and the Environment, 5(10): 540-548.

Lester S, Costello C, Halpern B, Gaines S, White C, Barth J. 2013. Evaluating trade-offs among ecosystem services to inform marine spatial Planning. Marine Policy, 38: 80-89.

Maguire B, Potts J, Fletcher S. 2012. The role of stakeholders in the marine planning process-Stakeholder analysis within the Solent, United Kingdom. Marine Policy, 36: 246-257.

Malczewski J. 1999. GIS and multicriteria decision analysis. New York John Wiley \& Sons, Inc.

Manwaring B, Orenstein S. Unpublished 2011. Principles for Stakeholder Involvement in Coastal and Marine Spatial Planning. EEUU: Udall Foundation's U.S. Institute for Environmental Conflict Resolution. 


\section{照 \\ COSTAS}

Martínez A, Malagón J. Unpublished 2014. Impacto económico y social del puerto de Cartagena.

MarViva. 2013. Ordenamiento Espacial Marino: Una Guía de Conceptos y Pasos Metodológicos. Fundación Marviva.

Millennium Ecosystem Assessment. 2005. Ecosystems and Human Well-being: Synthesis. Washington DC: Island Press.

[MinAmbiente] Ministerio de Ambiente y Desarrollo Sostenible, [Cardique] Corporación Autónoma Regional del Canal del Dique, [DIMAR] Dirección General Marítima, [ANT] Agencia Nacional de Tierras, Alcaldía de Cartagena, [PNN] Parques Nacionales Naturales de Colombia, [Carsucre] Corporación Autónoma Regional de Sucre. 2016. Plan de manejo Área Marina Protegida de los Archipiélagos del Rosario y de San Bernardo. Colombia.

Ministerio de Ambiente, Vivienda y Desarrollo Territorial. 2005. Resolución $\mathrm{N}^{\circ} 0679$ del 31 de mayo de 2005. Bogotá (Colombia): Diario Oficial No. 45.927.

Ministerio de Defensa Nacional. 1984. Decreto Ley $N^{\circ}$ 2324/1984. Bogotá (Colombia).

Ministerio del Medio Ambiente. 2001. Política nacional ambiental para el desarrollo sostenible de los espacios oceánicos y las zonas costeras e insulares de Colombia. Bogotá (Colombia).

[MMO] Marine Management Organization. 2013. Potential for co-location of activities in marine plan areas. East Anglia (United Kingdom): Marine Management Organization.

Naciones Unidas. 2011. Resolución N65/37 17 de marzo de 2011.

[OECD] Organisation for Economic Cooperation and Development. 2020. Sustainable Ocean for All: Harnessing the Benefits of Sustainable Ocean Economies for Developing Countries. The Development Dimension. Paris: OECD Publishing.

Osorio-Gómez J, Orejuela-Cabrera J. 2008. El proceso de análisis jerárquico (AHP) y la toma de decisiones multicriterio, ejemplo de aplicación. Scientia ET Technica, 14(39): 247-252.

Ouchi F. 2004. A literature review on the use of expert opinion in probabilistic risk analysis - Policy Research Working Paper 3201. Washington (EEUU): World Bank.

Papadimitriou A, Pangalos K, Duvaux-Béchon I, Giannopapa C. 2019. Space as an enabler in the maritime
Revista Costas, Vol. Esp. 2, 2021

sector. Acta Astronautica, 162: 197-206.

Papageorgiou M. 2016. Coastal and marine tourism: A challenging factor in Marine Spatial Planning. Ocean \& coastal management, 129: 44-48.

Portman M. E. 2016. Environmental Planning for Oceans and Coasts - Methods, Tools and Technologies. Switzerland: Springer International Publishing. $15 \mathrm{Vol}$.

Portman ME, Dalton TM, Wiggin J. 2015. Revisiting Integrated Coastal Zone Management: Is It Past Its Prime?. Environment: Science and Policy for Sustainable Development, 57 (2): 28-37.

Prato J, Reyna J. 2015. Aproximación a la valoración económica de la zona marina y costera del Caribe colombiano. Secretaría Ejecutiva de la Comisión Colombiana del Océano. Bogotá: Comisión Colombiana del Océano.

Pujos M, Pagliardini J.L, Steer R, Vemette G, Weber O. 1986. Influencia de la contra-corriente norte colombiana para la circulación de las aguas en la plataforma continental su acción sobre la dispersión de los efluentes en suspensión del río Magdalena. Boletín Científico CIOH, 6: 3-15.

Regan H, Davis F, Andelman S, Widyanata A, Freese M. 2007. Comprehensive criteria for biodiversity evaluation in conservation planning. Biodiversity and Conservation, 16: 2715-2728.

Schultz-Zehden A, Weig B, Lukic I. 2019. Maritime Spatial Planning and the EU's Blue Growth Policy: Past, Present and Future Perspectives. In: Zaucha J, Gee K, editors. Maritime Spatial Planning past, present, future. Cham (Switzerland): Springer International Publishing AG. p. 121 - 150.

Steer R, Arias-Isaza F, Ramos A, Sierra-Correa P, Alonso D, Ocampo P. 1997. Documento base para la elaboración de la "Política Nacional de Ordenamiento Integrado de las Zonas Costeras Colombianas". Documento de consultoría para el Ministerio del Medio Ambiente. Colombia: Acta Nocturna E.U.

Stelzenmüller V, Lee J, South A, Foden J, Rogers S. 2013. Practical tools to support marine spatial planning: A review and some prototype tools. Marine Policy, 38: 214-227.

Trenkamp R, Kellogg J, Freymueller J, Mora H. 2002. Wide plate margin deformation, southern Central America and northwestern South America, CASA GPS observations. Journal of South American Earth Sciences, 15: 157-171. 


\section{Afanador Franco et al.}

Tsilimigkas G, Rempis N. 2017. Maritime spatial planning and spatial planning: Synergy issues and incompatibilities. Evidence from Crete Island, Greece. Ocean \& coastal management, 139: 33-41.

Tuda A, Stevens T, Rodwell L. 2014. Resolving coastal conflicts using marine spatial planning. Journal of Environmental Management, 133: 59-68.

[UNEP] United Nations Environment Programme. 2015. Blue Economy: Sharing Success Stories to Inspire Change. GRID - Arendal. Norway.

[UNESCO] Intergovernmental Oceanographic Commission and United Nations Educational, Scientific and Cultural Organization. 2014. Indicadores UNESCO de cultura para el desarrollo - Manual metodológico. Paris (France): UNESCO.

[UNESCO] United Nations Educational, Scientific and Cultural Organization. 2017. World-wide Status and Trends of Maritime/Marine Spatial Planning - Presented at the 2nd International Conference on $\mathrm{Ma}$ rine/Maritime Spatial Planning. Paris (France): Motionous.

Vallega A. 1999. Fundamentals of integrated coastal management. Genoa (Italia): Springer Science \& Business Media Dordrecht. 49 Vol.

\section{re \\ COSTAS}

Villa F, Tunesi L, Agardy T. 2002. Zoning marine protected areas through spatial multiple-criteria analysis: the case of the Asinara Island National Marine Reserve of Italy. Conservation Biology, 16(2): 515-526.

Yatsalo B, Kiker G, Kim J, Bridges T, Seager T, Gardner K, Satterstrom K, Linkov I. 2007. Application of Multicriteria Decision Analysis Tools to Two Contaminated Sediment Case Studies. Integrated Environmental Assessment and Management. 3(2): 223-233.

Zervaki A. 2018. The Ecosystem Approach and Public Engagement in Ocean Governance: The Case of Maritime Spatial Planning. In: Langlet D and Rayfuse R, editors. The Ecosystem Approach in Ocean Planning and Governance - Perspectives from Europe and Beyond. The Netherlands: Koninklijke Brill NV, Leiden. 87 Vol. p. 223-255.

Zevallos-León. 2008. Operación y mantenimiento de una red de cable submarino [dissertation]. [Lima (Perú)]: Pontificia Universidad Católica del Perú. 


\section{부름 \\ COSTAS}

Revista Costas, Vol. Esp. 2, 2021

$>1644$ 